\title{
Orbital tumours and tumour-like lesions: exploring the armamentarium of multiparametric imaging
}

\author{
Bela S. Purohit ${ }^{1} \cdot$ Maria Isabel Vargas $^{2} \cdot$ Angeliki Ailianou $^{1} \cdot$ Laura Merlini $^{1}$ • \\ Pierre-Alexandre Poletti ${ }^{1}$ • Alexandra Platon ${ }^{1}$ • Bénédicte M. Delattre ${ }^{1}$. Olivier Rager $^{3}$. \\ Karim Burkhardt ${ }^{4} \cdot$ Minerva Becker ${ }^{1}$
}

Received: 1 September 2015 / Revised: 3 October 2015 / Accepted: 8 October 2015 /Published online: 31 October 2015

(C) The Author(s) 2015. This article is published with open access at Springerlink.com

\begin{abstract}
Although the orbit is a small anatomical space, the wide range of structures present within it are often the site of origin of various tumours and tumour-like conditions, both in adults and children. Cross-sectional imaging is mandatory for the detection, characterization, and mapping of these lesions. This review focuses on multiparametric imaging of orbital tumours. Each tumour is reviewed in relation to its clinical presentation, compartmental location, imaging characteristics, and its histological features. We herein describe orbital tumours as lesions of the globe (retinoblastoma, uveal melanoma), optic nerve sheath complex (meningioma, optic nerve glioma), conal-intraconal compartment (hemangioma), extraconal compartment (dermoid/epidermoid, lacrimal gland tumours, lymphoma, rhabdomysarcoma), and bone and sinus compartment (fibrous dysplasia). Lesions without any typical compartmental localization and those with multicompartment involvement (veno-lymphatic malformation, plexiform neurofibroma, idiopathic orbital pseudotumour, IgG4 related disease, metastases) are also reviewed. We
\end{abstract}

Minerva Becker

Minerva.Becker@hcuge.ch

1 Department of Radiology, Geneva University Hospital and University of Geneva, RueGabrielle-Perret-Gentil 4, 1211, Geneva 14, Switzerland

2 Department of Neuroradiology, Geneva University Hospital and University of Geneva, RueGabrielle-Perret-Gentil 4, 1211, Geneva 14 , Switzerland

3 Department of Nuclear Medicine, Geneva University Hospital and University of Geneva, RueGabrielle-Perret-Gentil 4, 1211, Geneva 14 , Switzerland

4 Department of Clinical Pathology, Geneva University Hospital and University of Geneva, RueGabrielle-Perret-Gentil 4, 1211, Geneva 14 , Switzerland discuss the role of advanced imaging techniques, such as MR diffusion-weighted imaging (DWI), diffusion tensor imaging, fluoro-2-deoxy-D-glucose positron emission tomography CT (FDG-PET CT), and positron emission tomography MRI (MRI PET) as problem-solving tools in the evaluation of those orbital masses that present with non-specific morphologic imaging findings.

Main messages/Teaching points

- A compartment-based approach is essential for the diagnosis of orbital tumours.

- CT and MRI play a key role in the work-up of orbital tumours.

- DWI, PET CT, and MRI PET are complementary tools to solve diagnostic dilemmas.

- Awareness of salient imaging pearls and diagnostic pitfalls avoids interpretation errors.

Keywords Orbit tumours · Magnetic resonance imaging (MRI) · Diffusion weighted imaging (DWI) · Positron emission tomography CT (PET CT) · Positron emission tomography MRI (MRI PET)

$\begin{array}{ll}\text { Abbreviations } \\ \text { ACC } & \text { Adenoid cystic carcinoma } \\ \text { ADC } & \text { Apparent diffusion coefficient } \\ \text { BMT } & \text { Benign mixed tumour } \\ \text { CECT } & \text { Contrast-enhanced CT } \\ \text { CEMRI } & \text { Contrast-enhanced MRI } \\ \text { DTI } & \text { Diffusion tensor imaging } \\ \text { DWI } & \text { Diffusion-weighted imaging } \\ \text { FA } & \text { Fractional anisotropy } \\ \text { FD } & \text { Fibrous dysplasia } \\ \text { FDG } & \text { Fluoro-2-deoxy-D-glucose } \\ \text { PET CT } & \text { positron emission tomography CT }\end{array}$




$\begin{array}{ll}\text { MRI PET } & \text { positron emission tomography MRI } \\ \text { FS } & \text { Fat saturated } \\ \text { FT } & \text { Full echo train } \\ \text { HR } & \text { High resolution } \\ \text { H\&E stain } & \text { Haematoxylin-eosin stain } \\ \text { IHC } & \text { Immunohistochemistry } \\ \text { IgG4-RD } & \text { Immunoglobulin G4-related disease } \\ \text { IOP } & \text { Inflammatory orbital pseudotumour } \\ \text { MALT } & \text { Mucosa associated lymphoid tissue } \\ \text { NECT } & \text { Non contrast-enhanced CT } \\ \text { NF } & \text { Neurofibromatosis } \\ \text { NHL } & \text { Non-Hodgkin's lymphoma } \\ \text { ONG } & \text { Optic nerve glioma } \\ \text { ONSM } & \text { Optic nerve sheath meningioma } \\ \text { OLPD } & \text { Orbital lympoproliferative disorders } \\ \text { OPNF } & \text { Orbital plexiform neurofibroma } \\ \text { RMS } & \text { Rhabomyosarcoma } \\ \text { RT } & \text { Radiotherapy } \\ \text { SCC } & \text { Squamous cell carcinoma } \\ \text { SUV } & \text { Standardised uptake value } \\ \text { US } & \text { Ultrasound } \\ \text { VLM } & \text { Veno-lymphatic malformation }\end{array}$

\section{Introduction}

The orbit is a small anatomical space with a wide range of important structures within. Tumours and tumour-like lesions often arise from these orbital contents and are a common indication for the radiological evaluation of the orbit in both adults and children. Cross-sectional imaging plays a vital role in the diagnosis and management of these lesions. Knowledge of the clinical presentation and patient's age helps to limit the differential diagnosis and to determine the appropriate imaging modality. Certain orbital pathologies such as retinoblastoma and rhabdomyosarcoma (RMS) are typically found in children, whereas malignant uveal melanoma, lymphoma, and inflammatory orbital pseudotumour (IOP) are seen in adults. Clinical symptoms such as extraocular muscle palsies, diplopia, visual impairment, exophthalmos, and eye pain can serve as useful pointers to the likely pathology. However, a biopsy may be needed to provide tissue diagnosis [1-4].

The purpose of this review is to discuss the clinical, imaging, and histopathological features of commonly encountered orbital tumours and tumour-like conditions. We highlight the role of cross-sectional imaging in the evaluation of indeterminate orbital masses with emphasis on advanced imaging techniques such as MR diffusion weighted imaging (DWI), diffusion tensor imaging (DTI), fluoro-2-deoxy-D-glucose (FDG)positron emission tomography CT (PET CT), and positron emission tomography MRI (PET MRI or MRI PET, the European Congress of Radiology uses MRI PET as the abbreviation to emphasize its imaging value). We discuss their impact on the differential diagnosis of those lesions, where conventional morphologic cross-sectional imaging is non-specific.

\section{Imaging techniques}

Evaluation of suspected orbital masses can be done with magnetic resonance imaging (MRI), computed tomography (CT), ultrasound (US), fluoro-2-deoxy-D-glucose positron emission tomography/CT (FDG-PET CT), and, recently, MRI PET. Most investigators agree that MRI is the imaging modality of choice, whereas CT should be reserved for cases with suspected bony pathology and whenever MRI cannot be performed. As shown by several investigators, high resolution MRI (HR MRI) shows superior soft tissue contrast than CT and allows more accurate depiction of the different orbital compartments [1-3]. MRI examinations can either be performed on a $1.5 \mathrm{~T}$ or on a $3 \mathrm{~T}$ scanner using a combination of head and surface coils. The standard MRI protocol in most institutions consists of axial SE T1W and TSE T2W sequences, coronal STIR sequences, and axial and coronal SE $\mathrm{T} 1 \mathrm{~W}$ sequences with fat saturation obtained after intravenous administration of gadolinium chelates. The recommended slice thickness is $2-3 \mathrm{~mm}$ with a $512 \times 512$ matrix. $3 \mathrm{D}$ FT heavily T2W sequences (CISS, FIESTA, or DRIVE) and 3DFT T1W sequences with $0.6-1 \mathrm{~mm}$ thin slices (VIBE, THRIVE) after intravenous contrast material administration are additionally used by some investigators including ourselves, as these volumetric data sets allow multiplanar reconstructions in any given plane, thereby facilitating the evaluation of subtle findings.

In patients with suspected orbital masses, $\mathrm{CT}$ is the modality of choice for evaluating calcifications and osseous orbital lesions and in patients with possible metallic foreign bodies [1-3]. CT examinations are usually performed using $0.6-$ $1 \mathrm{~mm}$ thin slices after intravenous injection of iodinated contrast material. Thin-slice HR multidetector $\mathrm{CT}$ provides quick volumetric acquisitions and precise depiction of the globe, optic nerve, intraconal, and extraconal spaces. Standard coronal and sagittal reconstructions are routinely obtained with bone and soft tissue settings.

Because of the superficial location of the globe and its cystic nature, ultrasound (US) with Doppler allows accurate depiction of a variety of pathologic conditions of the globe and orbit, and enables non-invasive and cost-effective followup. The technique is well tolerated, easy to perform, and has a high accuracy for the characterization of vascular lesions in skilled hands.

\section{Role of advanced imaging}

In recent years, advanced imaging techniques such as DWI, DTI, FDG-PET CT, and MRI PET are being increasingly used 
in the pre-therapeutic work-up and post-therapeutic monitoring of patients with head and neck tumours [5-15]. These modalities are also making significant inroads in ophthalmologic imaging. The physiological and functional information obtained by these techniques can be used to complement morphological findings obtained from conventional imaging, thereby aiding non-invasive tissue characterization [4, 8-23]. Although DWI sequences are widely used to assess primary or recurrent head and neck tumours, their applications in the orbit are still limited in clinical routine due to geometric distortion caused by the adjacent bone, air, and soft tissue interfaces. Nevertheless, the development of newer, robust single shot echo-planar DWI or multishot echo-planar DWI (such as the RESOLVE sequence) holds promise for the evaluation of orbital tumours and optic nerve pathology. DWI produces contrast on the basis of diffusivity of water molecules in different tissues and can thus be a source of information for assessing pathological tissue. Echo-planar DWI can help to differentiate between benign and malignant orbital masses based on apparent diffusion coefficient (ADC) values. DWI is more useful in characterizing infiltrative orbital masses, which appear hypointense on T2W images as compared to well-defined hyperintense lesions. Malignant masses especially orbital lymphomas show visually and quantitatively lower ADC values as compared to benign masses. Restricted diffusion is attributed to the higher cellularity and higher nuclear: cytoplasmic ratios in malignant lesions. Sepahdari et al have reported that an ADC value of $1.0-1.15 \times 10^{-3} \mathrm{~mm}^{2} / \mathrm{s}$ represents an optimal threshold for predicting malignancy with a reported sensitivity of $95 \%$, specificity of $91 \%$ and accuracy of $93 \%[9$, 10]. Visual assessment of benign and malignant lesions may not reveal significant differences on just DW images because of the T2-shine through effect. However, ADC maps are only affected by diffusivity changes. DWI can help to localize malignant masses in a background of non-specific inflammation and guide biopsy or intervention [9-13].

DTI is a novel MRI technique, which allows anatomic mapping and quantitative characterization of white matter architecture by measuring molecular diffusion [24]. DTI tractography is a computational procedure, which reconstructs white matter tracts in 3D-space based on their anisotropy characteristics. Tractography is used to provide a roadmap for the pre-surgical assessment of white matter tracts involved by tumours. Advances in MR data acquisition and postprocessing now permit high-resolution DTI of cranial nerves in a clinical setting. The antero-posterior orientation and fairly large size of the optic nerve and tracts provide favourable parameters for tractography. DTI can be used to map optic nerve fibres when involved by tumourous conditions such as gliomas or meningiomas. Depending on whether the tumour displaces or infiltrates the nerve fibres, appropriate functionsaving nerve surgery can be planned [3, 14-16]. However, DTI images of the optic nerve may show severe geometric distortion, in particular in the region of the orbital apex, and fibre tracking of the optic nerve is still part of ongoing clinical research protocols.

FDG-PET CT has recently emerged as a problem-solving tool in ophthalmologic oncology. It provides functional information regarding tumour metabolism based on FDG uptake. The level of FDG uptake depends upon tumour type and grade, size, surrounding metabolic activity, and blood glucose levels. For most primary orbital lesions, PET CT does not seem to provide any significant advantage over clinical evaluation and CT/MR imaging, other than detecting distant and metastatic lesions missed by conventional imaging. However, PET CT is now part of standard protocol in the initial staging work-up and post treatment assessment of orbital/ocular adnexal lymphomas. PET/CT also seems appropriate for metastatic tumours to the orbit and ocular adnexae from other primary sites. In these cases, one single comprehensive study may be preferable to performing multiple CT scans with contrast. The potential pitfalls in FDG-PET CT imaging of the orbit include background physiological uptake in extra-ocular muscles, poor uptake in very small lesions, and the likelihood of missing subtle uptake due to being at the edge of the field of view in standard skull to upper thigh PET CT scans [21]. Comparing standardized uptake values (SUV) between malignant and benign lesions and lesion to surrounding tissue helps in increasing diagnostic accuracy [17-21].

MRI PET leverages the superior soft-tissue contrast and functional sequences of MRI with the molecular information of PET in a single hybrid imaging technique. MRI PET is still a clinical research tool, however, recent publications have shown that MRI PET has an equivalent performance with respect to lesion detection as PET CT in the evaluation of head-neck cancers [7, 23]. Although large-scale data and studies on the role of MRI PET in orbital conditions are still awaited, it can be a considered as a potential future tool in refining orbital imaging. Paediatric and pregnant patients may benefit from MRI PET because of reduced radiation burden as compared to PET CT. MRI PET may play a valuable role in the evaluation of paediatric lymphomas, neuroblastomas. and soft tissue sarcomas [22, 23].

\section{Compartment-based approach to orbital masses}

The orbit can be anatomically divided into some well-defined compartments (Fig. 1). The muscle cone comprising the four rectus muscles divides the orbit into the intraconal and extraconal compartments. The intraconal compartment contains the globe, the optic nerve-sheath complex, orbital vessels. and nerves (Fig. 1). The extraconal compartment consists of the bony orbital walls, fat. and the lacrimal gland. The orbital septum and lid form the anterior or preseptal compartment. Localizing orbital lesions to these specific 

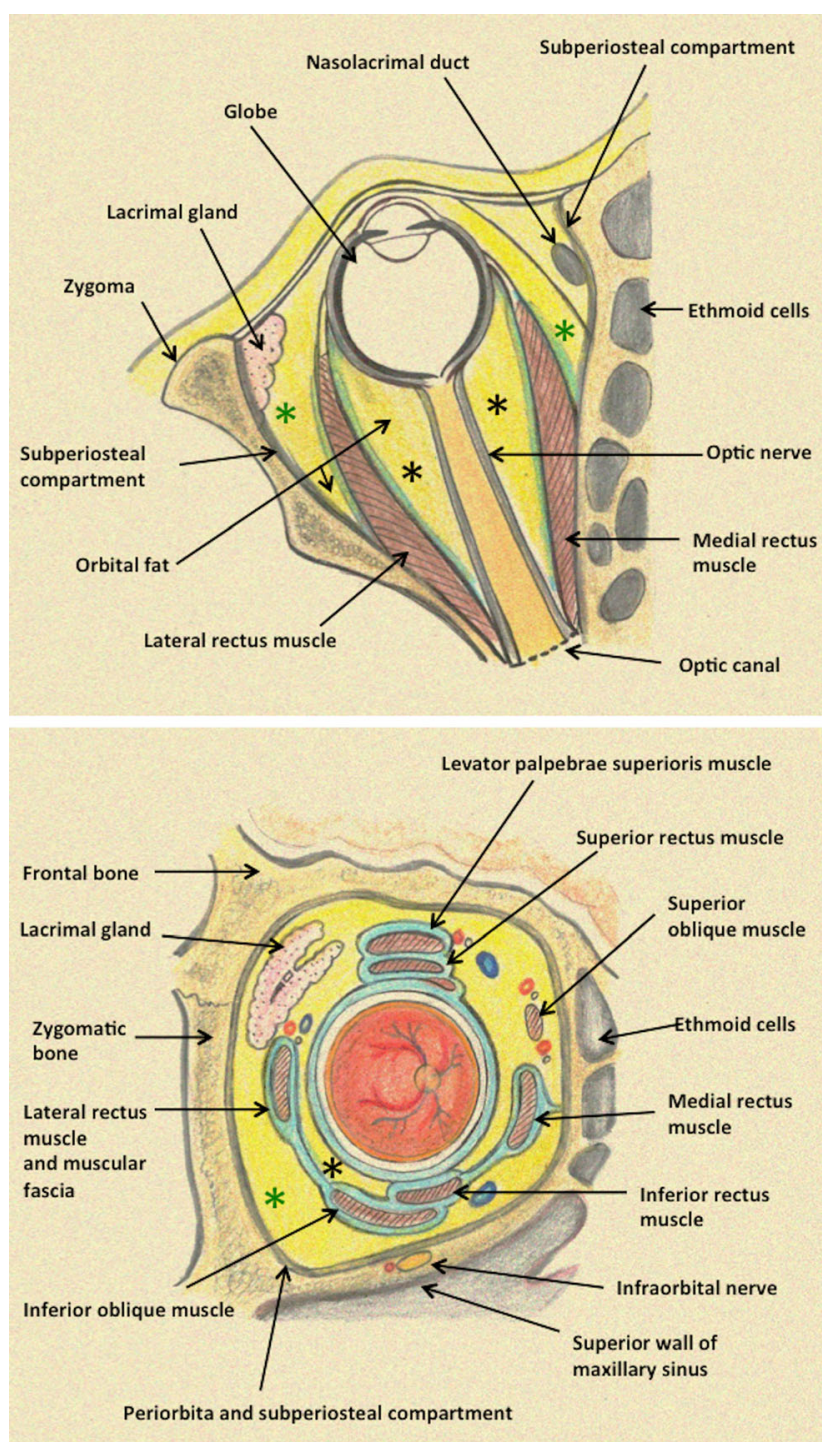

Fig. 1 Schematic illustration of the orbital contents and compartments. Axial and coronal view of the right orbit. Black asterisks indicate the intraconal compartment. Green asterisks indicate the extraconal compartment

compartments helps to simplify the diagnostic approach and narrow down the list of differentials [1].

In this review, we will describe tumours of the globe and optic nerve first, followed by the conal-intraconal tumours, extraconal tumours, and finally tumours of the bones/sinuses (Table 1). Examples of multi-compartmental masses will also be discussed (Table 1).

\section{Globe}

\section{Retinoblastoma}

Retinoblastoma is a malignant, primary retinal neoplasm and the most common intraocular tumour of childhood. About $90 \%$ of cases occur under 5 years of age. Retinoblastoma can occur in a hereditary form (40\%) or in a sporadic form $(60 \%)$. In both cases, patients have mutations of the retinoblastoma tumour suppressor gene on chromosome 13q14 [1, $3,4,25,26]$. Hereditary retinoblastoma is associated with early onset disease; screening ultrasound (US) and MRI have been recently recommended for the management of fetuses at high risk of developing this tumour [27]. The higher incidence of retinoblastoma seen in developing countries has been partly attributed to the presence of human papilloma virus in tumour tissue [28]. About $50 \%$ of cases present with leukocoria and $40 \%$ show bilateral disease. Although the initial diagnosis is based on ophthalmoscopy and US, cross-sectional imaging is mandatory to assess disease extent and prognosis. Extraocular extension occurs in less than $10 \%$ cases and portends poor prognosis. When a pinealoblastoma is associated with bilateral retinoblastomas, the term trilateral retinoblastoma is applied. Low-grade intra-ocular retinoblastomas are treated with chemotherapy while advanced tumours require enucleation and/or radiotherapy (RT) [1, 3, 4, 25, 26].

Retinoblastoma arises from precursor cells of the retinal neuroepithelium. Histologically, undifferentiated areas of the tumour show characteristic "small blue cells" with scant cytoplasm and large hyperchromatic nuclei. Differentiated structures called Flexner-Wintersteiner rosettes are commonly seen. Because of rapid growth of the tumour, necrosis and calcifications are common; these features account for the characteristic radiologic appearances on CT and MRI [25, 26]. Non-contrast-enhanced CT (NECT) demonstrates intratumoural calcifications in about $90 \%$ cases. Marked enhancement of the tumour is seen on contrast-enhanced CT (CECT). MRI of the orbits and brain is usually performed together to determine extraocular and intracranial extension as well as to rule out an associated pinealoblastoma. The tumour is slightly hyperintense on T1W images, hypointense on T2W images, and shows marked post contrast enhancement on contrastenhanced MRI (CEMRI) (Fig. 2) [1, 4, 25, 29]. Although $\mathrm{CT}$ is superior in calcium detection, MRI may allow equally accurate identification of calcifications by revealing spots of low signal intensity on very thin T2 TSE and T2* weighted sequences [30]. Retinoblastoma may exhibit variable hyperintensities on DWI resulting in varying values on ADC maps, depending on cellularity and histology. De Graaf et al have reported statistically significant differences in ADC values in viable enhancing tumour tissue $\left(1.03 \times 10^{-3} \mathrm{~mm}^{2} /\right.$ s) as compared to non-viable necrotic tissue $\left(1.47 \times 10^{-3} \mathrm{~mm}\right.$ $2 / s)$. Thus ADC values can be used for monitoring treatment response in retinoblastoma [31].

Other diseases causing leukocoria can be differentiated from retinoblastoma based on clinical presentation and imaging characteristics. Coat's disease, persistent primary hyperplastic vitreous and toxocariasis lack calcifications. Retinopathy of prematurity rarely shows calcifications, it may be bilateral and history of prematurity is usually elicited $[4,25,26]$. 

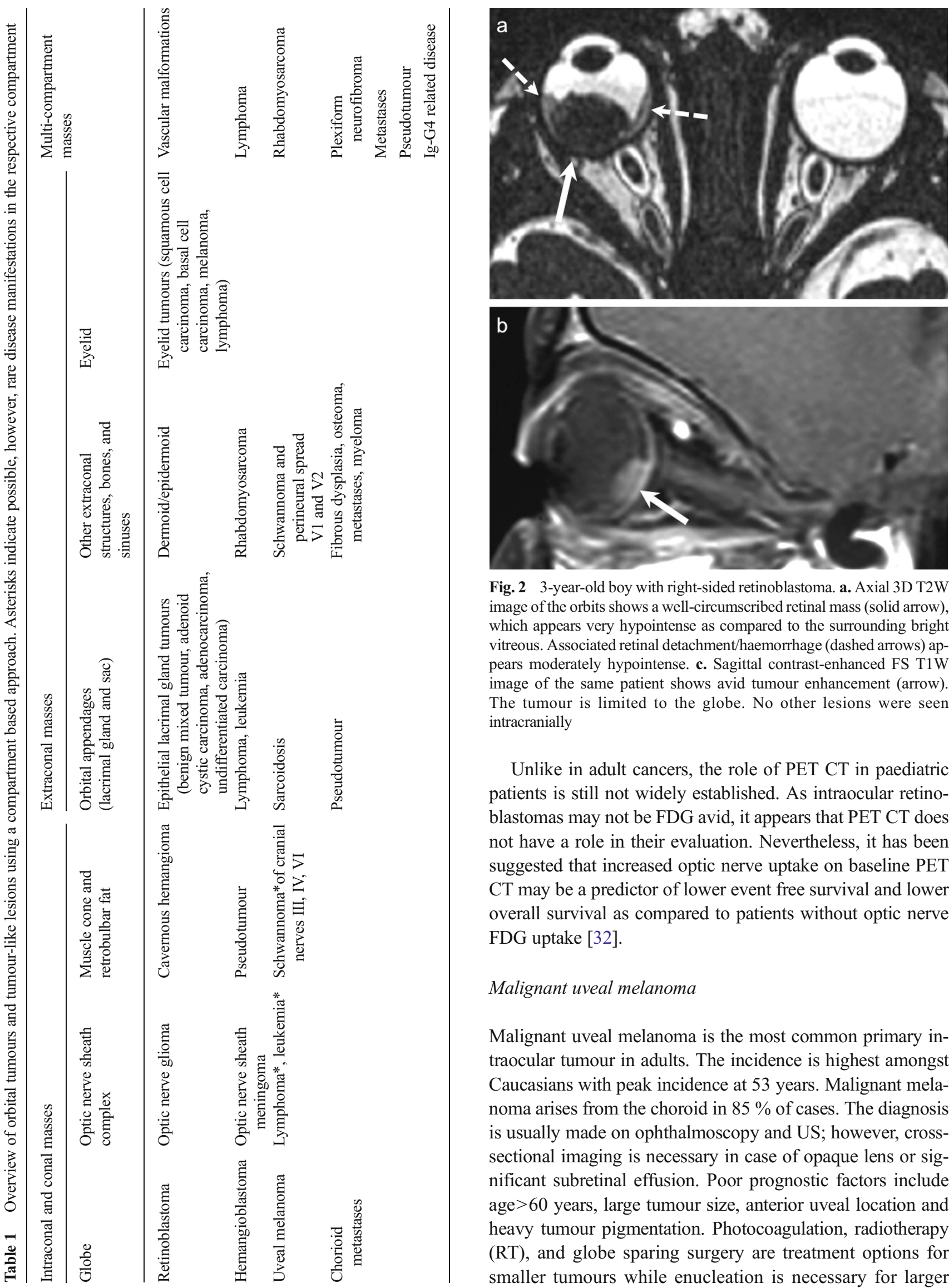

Fig. 2 3-year-old boy with right-sided retinoblastoma. a. Axial 3D T2W image of the orbits shows a well-circumscribed retinal mass (solid arrow), which appears very hypointense as compared to the surrounding bright vitreous. Associated retinal detachment/haemorrhage (dashed arrows) appears moderately hypointense. c. Sagittal contrast-enhanced FS T1W image of the same patient shows avid tumour enhancement (arrow). The tumour is limited to the globe. No other lesions were seen intracranially

Unlike in adult cancers, the role of PET CT in paediatric patients is still not widely established. As intraocular retinoblastomas may not be FDG avid, it appears that PET CT does not have a role in their evaluation. Nevertheless, it has been suggested that increased optic nerve uptake on baseline PET CT may be a predictor of lower event free survival and lower overall survival as compared to patients without optic nerve FDG uptake [32].

\section{Malignant uveal melanoma}

Malignant uveal melanoma is the most common primary intraocular tumour in adults. The incidence is highest amongst Caucasians with peak incidence at 53 years. Malignant melanoma arises from the choroid in $85 \%$ of cases. The diagnosis is usually made on ophthalmoscopy and US; however, crosssectional imaging is necessary in case of opaque lens or significant subretinal effusion. Poor prognostic factors include age $>60$ years, large tumour size, anterior uveal location and heavy tumour pigmentation. Photocoagulation, radiotherapy (RT), and globe sparing surgery are treatment options for smaller tumours while enucleation is necessary for larger 
tumours. Systemic metastases to the liver, lungs, and brain are often the cause of mortality [18, 26, 33].

The current WHO classification of choroidal tumours consists of three histological types, i.e. mixed, epitheloid, and necrotic melanomas. Spindle cell A tumours are now classified as benign choroidal nevi. The mixed type includes spindle $\mathrm{B}$ and epitheloid cells. The prognosis of spindle cell tumours is better as compared to mixed or purely epitheloid melanomas. Metastatic potential is determined by maximum tumour dimensions, number of epithelioid cells, vascular patterns, and nucleolar size and activity [26, 33, 34].

NECT shows a well-circumscribed, hyperdense, mushroom-shaped tumour with a broad choroidal base. Calcification is rare. CECT and CEMRI show marked post contrast enhancement (Fig. 3). The melanin content causes characteristic hyperintense signal on $\mathrm{T} 1 \mathrm{~W}$ and hypointense signal on T2W MR images. Thin-section MRI enables accurate prediction of the degree of melanomatous pigmentation based on quantitative evaluation of $\mathrm{T} 1 \mathrm{~W}$ images. More pronounced pigmentation is associated with poorer prognosis. Fat-saturated, high-resolution CEMRI is ideal for demonstrating small enhancing tumours, associated retinal detachment as well as extraocular disease. Imaging differentials include choroidal hemangioma, choroidal detachment, and uveal metastases [33]. False positive or false negative assessments for ocular melanoma and other ocular tumours may be caused by retinal detachment and highly myopic patients. In highly myopic patients, the globe can be elongated and the resulting chemical shift artefact can lead to asymmetric thickening of the outline

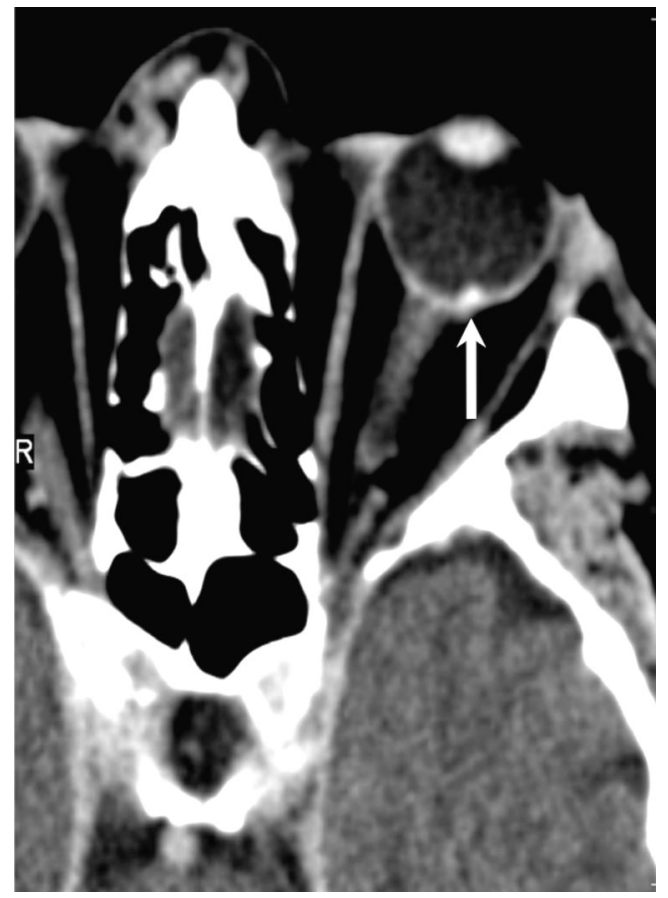

Fig. 3 50-year-old male patient diagnosed with a left-sided uveal melanoma on ophthalmoscopy. a. Axial contrast-enhanced CT image shows a tiny, avidly enhancing nodule (arrow) along the choroid of the globe mimicking the presence of a tumour while, on the contrary, retinal detachment may mask small tumours. ErbEigner et al have reported that ocular melanomas show marked restricted diffusion with a mean ADC value of $0.891 \times 10^{-3} \mathrm{~mm}^{2} / \mathrm{s}$. Also, the mean ADC of ocular melanoma appears to be statistically significant from the mean ADC of retinal detachment [35] thereby facilitating diagnosis.

FDG-PET CT has the potential to provide substantial benefit in the surveillance of uveal melanomas. Reddy et al found that PET CT was able to detect metabolic activity of choroidal melanomas, especially T3 and larger lesions. A positive correlation was found between SUV and the size of the melanoma. However, PET CT is unable to differentiate between small melanomas and suspicious choroidal nevi [36]. Whole body PET CT has been shown to play a valuable role in detecting regional and distant metastases from choroidal melanoma. Kurli et al have reported $100 \%$ sensitivity and specificity of PET CT in the identification of hepatic metastases of choroidal melanomas [37].

\section{Optic nerve-sheath complex}

\section{Optic nerve glioma $(O N G)$}

ONG is the most common primary optic nerve tumour. The low-grade form is commonly seen in children with $75 \%$ of cases presenting before the age of 10 years. The less common aggressive form is seen in adults and is often fatal. About $38 \%$ of paediatric patients with ONG have neurofibromatosis (NF)1 , and about $50 \%$ of patients with NF1 harbour ONG. Bilateral disease is pathognomonic of NF-1 (Fig. 4). Most paediatric ONG cases involve the intraorbital and intracranial portions of the optic nerve; about $20 \%$ may extend up to the chiasm, hypothalamus, and optic tracts. Involvement of the chiasm is common in adult ONG. Patients typically present with decreased vision and painless proptosis. Hypothalamic lesions can cause hydrocephalus. Benign ONG are slow growing tumours that do not metastasize. Imaging is mandatory to guide management and to document disease progression. The role of chemotherapy and radiotherapy (RT) in the treatment is still controversial. Long-term prognosis is excellent if the tumour is confined to the optic nerve; however, intracranial extension is associated with poorer survival rates $[1,4,8$, $25,26]$.

Most ONG are low-grade pilocytic astrocytomas (WHO grade1). Histologically, these tumours are biphasic and consist of bipolar cells and multipolar cells. Bipolar cells show pilocytic processes, whereas multipolar cells may show eosinophilic granular bodies. In addition, Rosenthal fibres, microcysts, and neovascularity are commonly seen. Adult forms are usually aggressive anaplastic astrocytoma (WHO grade 3 ) or glioblastoma multiforme (WHO grade 4) $[25,26]$. 


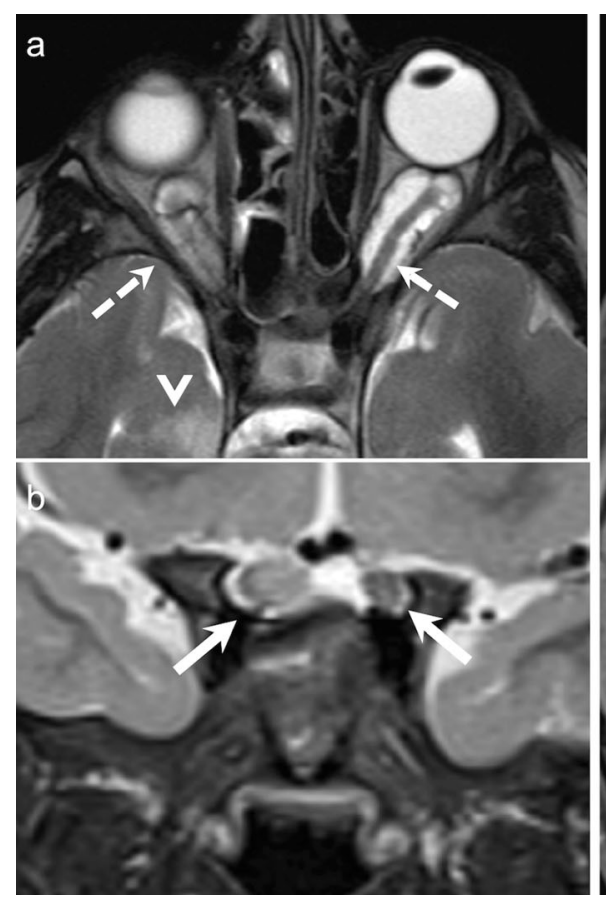

Fig. 4 5-year-old boy with NF-I. a. Axial T2W MR image of the orbits shows bilateral ONG (dashed arrows) causing fusiform enlargement and kinking of the optic nerves. A focal T2- hyperintense lesion (arrowhead) is seen in the right mesial temporal lobe. b. Coronal T2WMR image of the same patient shows extension of the bilateral ONG along the intracranial segments of the optic nerves (arrows). c. Axial contrast-

On CT and MRI, ONG show fusiform or tubular enlargement often with kinking of the optic nerve and ectasia of the optic sheath (Fig. 4). Calcification is rare. Widening of the optic canal and cystic degeneration may be seen. ONG are usually hypointense on $\mathrm{T} 1 \mathrm{~W}$ images, slightly hyperintense on $\mathrm{T} 2 \mathrm{~W}$ images and show variable enhancement (Fig. 4). MRI is the imaging modality of choice as it detects small tumours and elegantly maps out intraorbital as well as intracranial extensions. ONG can be differentiated from optic nerve sheath meningioma (ONSM) as the latter are commoner in adults, dark on $\mathrm{T} 2 \mathrm{~W}$ images, surround the optic nerve, and show avid post-contrast enhancement [1, 4, 8, 25, 26].

ONG show increased diffusion on DWI; they exhibit high $\mathrm{ADC}$ and low fractional anisotropy (FA) values. This is attributed to their low cellularity and low proliferative indices [11, $13,38,39]$. Jost et al have reported on the use of DWI and dynamic contrast-enhanced imaging to calculate the diffusivity and permeability of ONG. Similar to other pilocytic astrocytomas, $\mathrm{ONG}$ show $\mathrm{ADC}$ values in the range of 1.2-2.09 $\times 10$ ${ }^{-3} \mathrm{~mm}^{2} / \mathrm{s}$. ADC values cannot distinguish between clinically stable and clinically aggressive ONG. On the other hand, clinically aggressive $\mathrm{ONG}$ are seen to show significantly higher permeability values than clinically stable tumours [38]. DTI tractography can be used in the presurgical evaluation of ONG by demonstrating integrity of the optic nerve in patients with resectable lesions. Tractography can demonstrate divergence

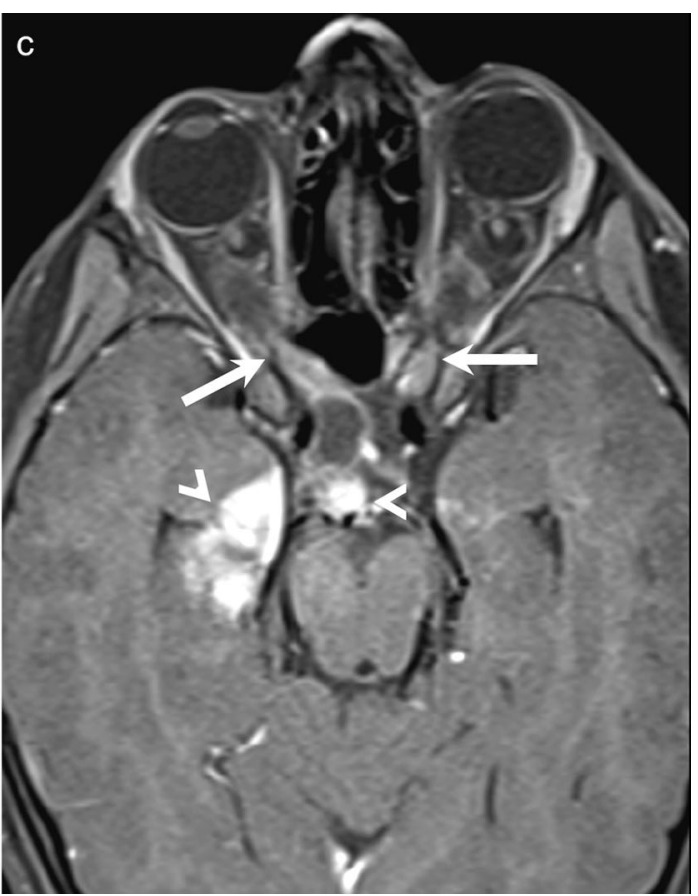

enhanced FS TIW MR image of the same patient shows prominent enhancement in the intracanalicular and intracranial segments of bilateral ONG (arrows). There is also avid enhancement in the suprasellar/chiasmatic region (arrowhead) and in the right mesial temporal lesion (arrowhead) in keeping with tumour extension along the optic chiasm and right optic radiation

or disruption of nerve fibres. This allows for minimal postsurgical morbidity and minimal vision loss [14-16, 39]. Fillipi et al found that DTI may offer increased sensitivity over conventional imaging in evaluating the optic pathways in NF-1 patients. These patients showed statistically significant decrease in FA values and elevated mean diffusivity values as compared to age-matched controls [40]. Currently, no reliable factors have yet been identified to predict future vision loss in patients with ONG. Nevertheless, de Blank et al found that decreased FA of the optic radiations in patients with ONG was predictive of visual acuity loss during the following year and, therefore, suggested that DTI may be used as a non-invasive tool to prospectively identify those patients who will require therapy [41].

Although PET CT is not used routinely for paediatric patients, Miyamoto et al have reported very high FDG uptake in an adult patient with ONG, which turned out to be an anaplastic astrocytoma on histology. They also reported an adult patient with fibrillary astrocytoma of the optic nerve showing isointense FDG uptake. Thus, PET CT may potentially play a role in the histological grading of ONG, especially in adult patients [19].

\section{Optic nerve sheath meningioma (ONSM)}

ONSM is the most common primary tumour arising from the optic nerve sheath. It is a benign, slow-growing tumour, which 
accounts for $5 \%$ of primary orbital tumours and $2 \%$ of all meningiomas. It commonly occurs in females between 30-70 years of age. Hormonal factors, radiation exposure, and hereditary predisposition (abnormalities of chromosome 22) have been implicated in the aetiology. It is rare in children except in cases of neurofibromatosis (NF)-2. Primary ONSM arise from the intraorbital and intracanalicular segments of the optic nerve while secondary ONSM are intraorbital extensions of intracranial tumours. A primary ONSM may extend intracranially to involve the contralateral optic nerve. ONSM typically presents with slowly progressive painless loss of vision (with preservation of central visual field) and progressive proptosis. Although benign, these tumours have a tendency to recur. Because of their slow progression, small ONSM are often managed just by conservative follow-up imaging. Fractionated stereotactic RT is the treatment of choice for patients with preservable vision $[1,8]$.

ONSM arises from the arachnoid cap cells within the optic nerve sheath. Histologically, an ONSM reveals various subtypes of which the meningothelial (lobules of tumour cells), fibrous (bundles of spindle cells), and transitional types (whorls, psammoma bodies) are most common. All these are WHO grade 1 benign tumours. In benign meningiomas, tumour cells are uniform, mitoses are rare and necrosis is absent. Atypical meningiomas (WHO grade 2) have increased mitotic activity and cellularity, prominent nucleoli, and necrosis; some are of clear cell subtype. Anaplastic meningiomas (WHO grade 3) include rhabdoid/papillary subtypes and histological features of frank malignancy like extensive mitoses and necrosis [26, 42].

The diagnosis of ONSM depends on cross-sectional imaging. NECT commonly shows tubular thickening and calcification of the optic nerve sheath complex. An enlarged optic nerve canal and hyperostosis may be seen. Although MRI is less sensitive than CT for detection of calcifications, it is ideal for assessing the intracanalicular and intracranial extension of ONSM. ONSM demonstrates similar intensity as the optic nerve on T1W and T2W images. Fat-saturated, thin-section CEMRI images show a tubular enhancing mass around the isointense optic nerve (tram track sign on axial images/target sign on coronal images) (Fig. 5). ONG is the closest imaging differential; however, ONG is more common in children, does not calcify, expands the optic nerve, often associated with other stigmata of NF-1, and may extend intracranially along the optic pathways $[1,8]$. Abnormal enhancement of the optic nerve sheath causing the tram track sign or the target sign appearance on cross-sectional imaging may also be seen in the setting of lymphoma, leukemia, and inflammatory pseudotumour or can be caused by tumour seeding into the subarachnoid space (as the optic nerve sheath communicates with the intracranial subarachnoid space).

Because of its hypercellular nature, ONSM often shows restricted diffusion with low ADC values on DWI [9-12]
(Fig. 5). Sepahdari et al have reported ADC values in the range of $0.79-1.04 \times 10^{-3} \mathrm{~mm}^{2} / \mathrm{s}$ for ONSM [9]. Zang et al have illustrated a case of tractography demonstrating optic nerve displacement and atrophy due to an invasive orbital meningioma [15].

It has been reported that WHO grades 2 and 3 dural meningiomas show higher FDG uptake than grade 1 tumours. Thus, FDG uptake correlates with the proliferative activity of meningiomas [43, 44].

\section{Conal-intraconal compartment}

\section{Cavernous hemangioma}

Cavernous hemangioma is the most common benign orbital tumour-like condition in adults. According to the classification of vascular malformations by the International Society for the Study of Vascular Anomalies (ISSVA), cavernous hemangioma is a venous or low flow malformation $[1-4,8]$. It is more common in women and usually seen in the 2 nd -4 th decades. It is typically intraconal and presents with slowly progressive, unilateral proptosis, and/or diplopia. Occasionally, compression of the optic nerve may result in mild visual deficit. Unlike childhood capillary hemagiomas, cavernous hemangiomas do not show cellular proliferation nor do they have a prominent arterial supply. In fact, they are isolated from the orbital vascular system. Asymptomatic tumours are usually observed conservatively. Surgical resection is performed to correct visual disturbance and cosmetic problems. Visual prognosis is excellent in most cases [1, 2, 45, 46].

Histologically, cavernous hemangiomas consist of large blood-filled spaces lined by flattened endothelial cells and separated by scant fibrous tissue. Stagnant circulation may result in venous thrombosis. Dystrophic calcification is common $[26,45]$. Cavernous hemangiomas can coexist with other vascular malformations of the orbit like venous varix and lymphangioma [47].

On NECT, a cavernous hemangioma is seen as a wellcircumscribed, dense intraconal mass. It is usually seen separately from the optic nerve and extraocular muscles CECT may show patchy or uniform enhancement (Fig. 6). Phleboliths, if present, help to confirm the diagnosis (Fig. 6). On MRI, the lesion typically appears iso- to hypointense on $\mathrm{T} 1 \mathrm{~W}$ images and hyperintense on $\mathrm{T} 2 \mathrm{~W}$ images, although some hemangiomas may display moderately high/low signal intensity. Some scattered hyperintense areas on T1W images may indicate thrombosis. Fat-suppressed, dynamic CEMRI shows initial patchy enhancement followed by homogeneous enhancement in the delayed phase (Fig. 7). Imaging differentials include venous varix and, less often, schwannoma. A venous varix shows intermittent intralesional flow and exophthalmos on Valsalva manoeuvre [1, 2, 45, 46]. Schwannomas also appear well-circumscribed, iso-hypointense on T1W 

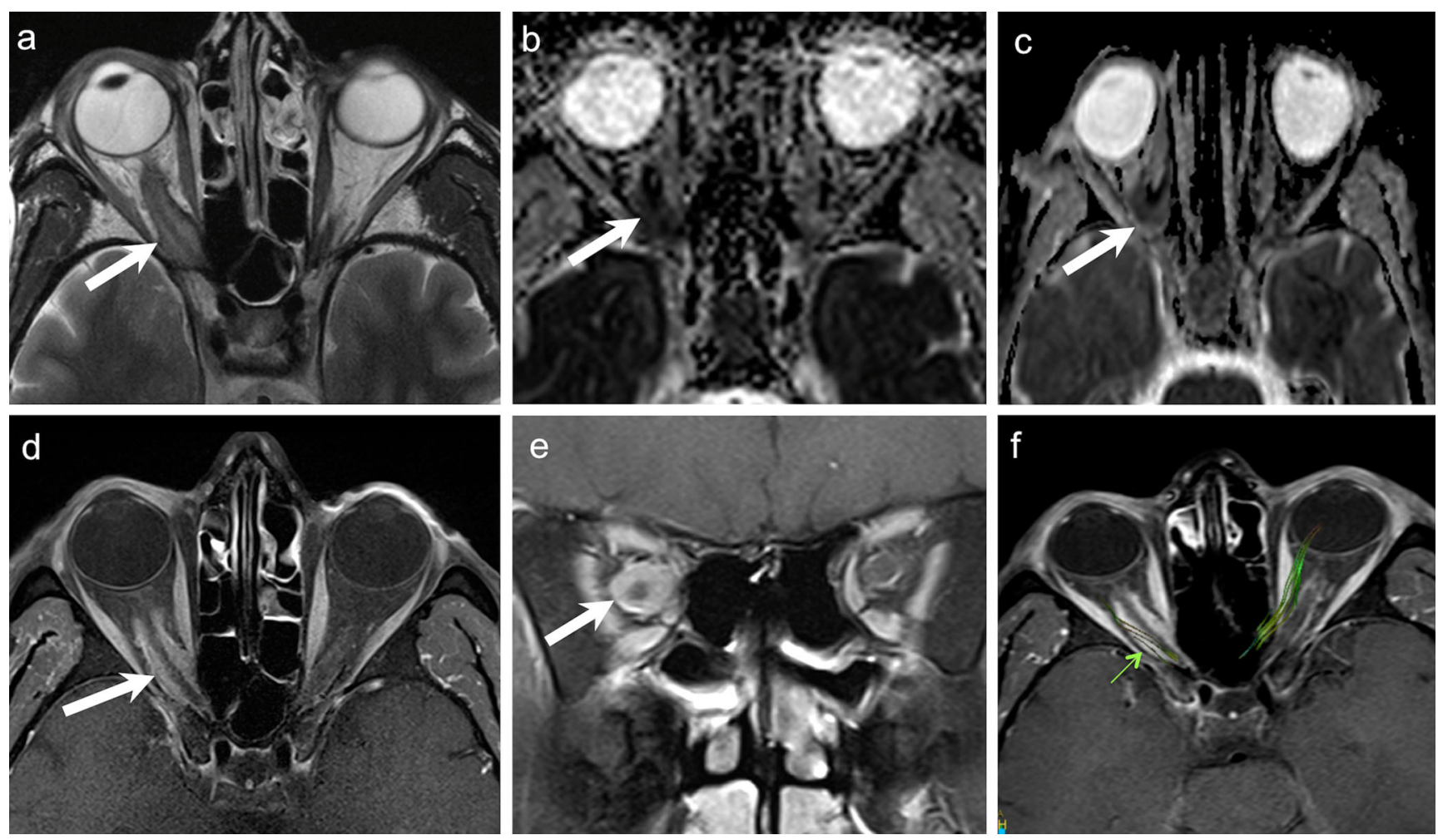

Fig. 5 41-year-old woman with right-sided progressive vision loss and proptosis. a. Axial T2W image shows a moderately hpointense fusiform lesion originating from the optic nerve sheath encasing the optic nerve (arrow). b. Corresponding axial ADC map from RESOLVE DWI shows restricted diffusion (arrow). Note only minor image deformation. c. ADC map from standard EPI sequence also shows restricted lesion diffusivity (arrow); however, note major image deformation due to susceptibility artefacts. d. Axial contrast enhanced T1W image reveals strong fusiform enhancement along the optic nerve ("tram track sign", arrow). There was no extension through the orbital apex intracranially. e. Coronal fat saturated $\mathrm{T} 1 \mathrm{~W}$ image displaying concentric enhancement of the tumour around the compressed optic nerve creating a characteristic "bull's eye" appearance (arrow). Imaging findings are in keeping with an ONSM. f. DTI 3D tractography reconstruction of the optic nerves reveals normal fibres on the left (fibers are depicted in green due to their anterior- posterior course) and major fibre atrophy on the right (arrow)
Fig. 6 Orbital hemangioma as seen on CT in two different patients. a. Coronal NECT in a 30 -year-old male patient shows a well-circumscribed intraconal mass (arrow) with calcified phleboliths. b-d. 50-year-old male patient with an incidentally diagnosed left orbital cavernous hemangioma on an angio-CT performed for stroke. $\mathbf{b}$. NCECT shows a nonspecific intraconal lesion (arrow) without phleboliths. Arterial phase (c) demonstrates initial patchy enhancement (arrow) followed by progressive filling of the lesion in the venous phase (d)
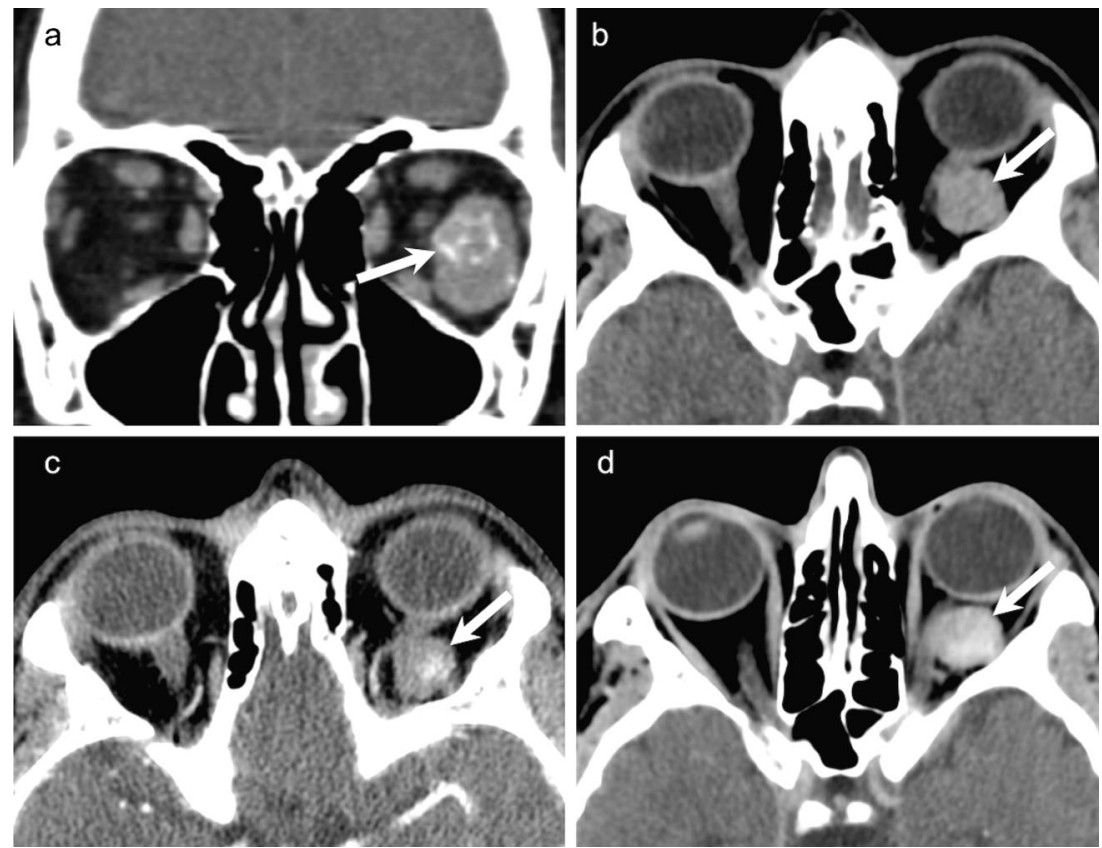

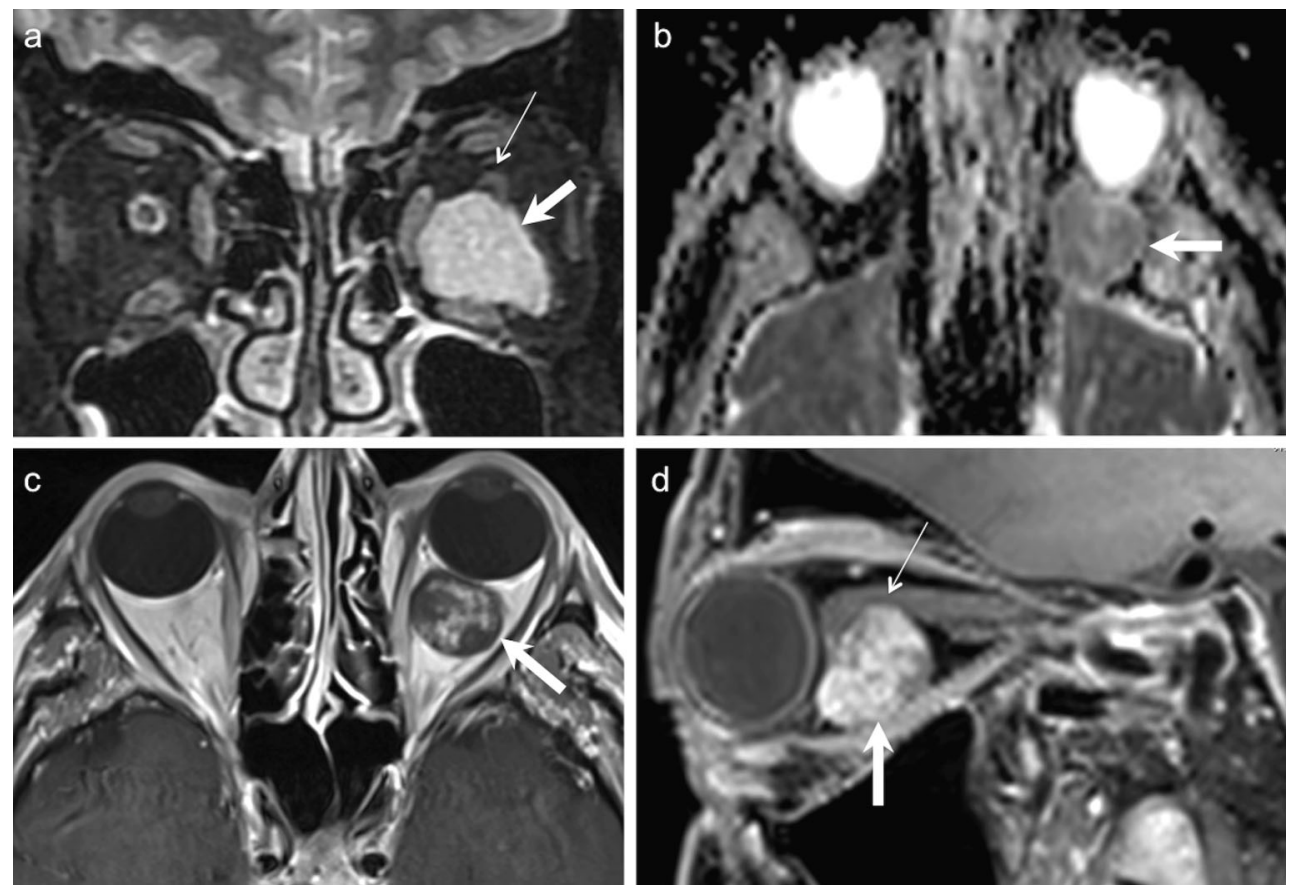

Fig. 7 60-year-old man with left orbital cavernous hemangioma. Coronal STIR image (a) shows a well-circumscribed hyperintense intraconal mass (arrow) causing superior displacement of the left optic nerve (thin arrow). b. ADC map shows moderately hypointense signal within the mass with an ADC value of $1.4 \times 10^{-3} \mathrm{~mm}^{2} / \mathrm{s}$. c. Axial contrast-enhanced TIW

image shows initial patchy enhancement. d. Sagittal reformatted image from a contrast-enhanced 3D VIBE acquisition obtained after the T1W sequence shows characteristic progressive filling (arrow). Major mass effect on the optic nerve (thin arrow). The patient underwent surgery, which confirmed the diagnosis of cavernous hemangioma

images, and hyperintense on $\mathrm{T} 2 \mathrm{~W}$ images. However, the spread pattern on dynamic CEMRI can help to distinguish between schwannoma and cavernous hemangioma. In the early phase, enhancement in hemangiomas starts at one point, whereas it starts from a wide area in schwannomas [48, 49].

Cavernous hemangiomas appear bright on DW images with mean $\mathrm{ADC}$ values ranging from $1.23 \times 10^{-3} \mathrm{~mm}^{2} / \mathrm{s}$ to $1.39 \times 10^{-3} \mathrm{~mm}^{2} / \mathrm{s}[9,10,12]$ (Fig. 7). Razek et al have reported that schwannomas have mean ADC value of $1.92 \times 10^{-3}$ $\mathrm{mm}^{2} / \mathrm{s}$, which is statistically different from that of cavernous hemangiomas [12].

Scinitigraphy using technetium 99 m-labelled red blood cells can help in the differential diagnosis of cavernous hemangioma from other imaging mimics [50]. Miyamoto et al have described two cases of adult orbital cavernous hemangiomas showing isointense FDG uptake on PET/CT [19].

\section{Extraconal compartment}

\section{Dermoid}

Dermoids are the most common congenital orbital lesions, accounting for about $25 \%$ of all orbital biopsies. They arise from epithelial sequestration, usually at the zygomaticofrontal and frontoethmoidal sutures. They are typically seen in the extraconal region, superolaterally, between the globe and the

orbital periosteum. They are slow growing lesions; however, they may occasionally rupture and mimic acute inflammation. Small dermoids do not require immediate treatment and are usually removed within the 2 nd or 3 rd year of life. Complete excision without rupturing is necessary in order to avoid inflammatory reactions or local recurrences $[1,26,51]$.

Macroscopically, orbital dermoids are round or ovalshaped, encapsulated tumours, containing various skin appendages and fatty material. Histologically, a dermoid is lined by squamous epithelium and may contain hair follicles, sebaceous glands as well as keratinous debris [26, 51].

In addition to clinical examination, which is sufficient for most superficial dermoids, US scans help to demonstrate a sharply outlined lesion with a capsule and low-reflectivity contents. CT or MRI are rarely necessary. NECT shows a well circumscribed, cystic tumour of low or fat density. Fat-fluid levels and calcifications may be seen. Bony scalloping of the lacrimal fossa may occur due to pressure effect. On MRI, dermoids typically appear hyperintense on $\mathrm{T} 1 \mathrm{~W}$ and $\mathrm{T} 2 \mathrm{~W}$ and hypointense on STIR (Fig. 8). Rim enhancement is rare $[1,26,51]$. Diffusion in dermoids may be variable depending upon their contents. Lope et al and Sepahdari et al have described restricted diffusion in orbital dermoids [9, 13], whereas Razek et al have described ADC values in the range of $1.68-1.95 \times 10^{-3} \mathrm{~mm}^{2} / \mathrm{s}$ for head-neck dermoids [52]. Most often, the detection of macroscopic fat in dermoids is diagnostic and DWI adds no additional value (Fig. 8). 

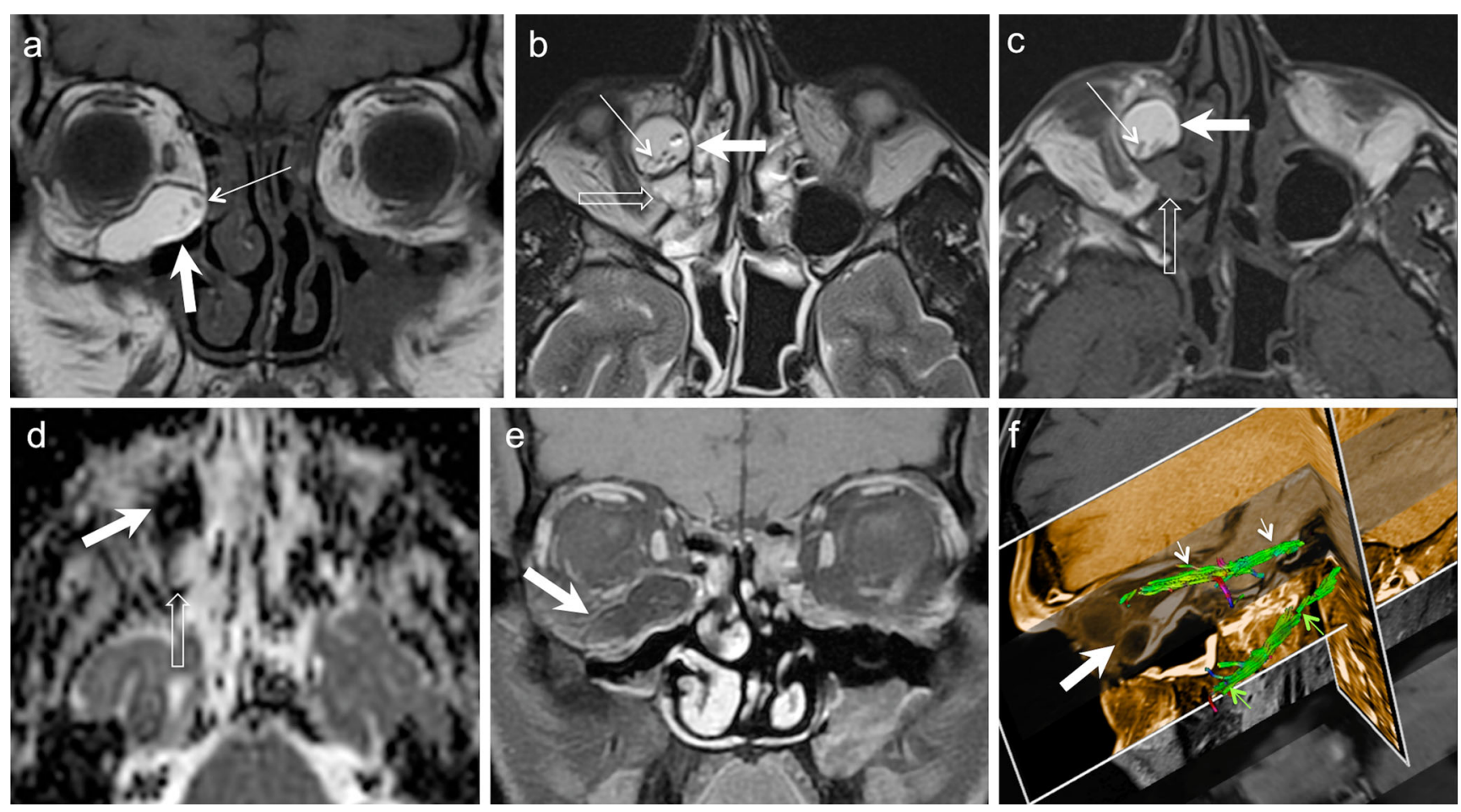

Fig. 8 42-year-old male with a histologically proven right orbital dermoid. Coronal T1W (a), axial T2W (b), axial T1W (c), axial ADC map (d), and coronal FS contrast-enhanced T1W (e) images show an orbital lesion with an anterior component containing fatty tissue (thick white arrows) and a posterior component containing non-fatty elements (hollow arrows). There are some fluid droplets in the anterior component (thin arrows). Note minor capsular enhancement after gadolinium

Cephalocoeles can occur in the fronto-orbital region near the midline and can mimic dermoids. Cephalocoeles show a track leading to the anterior cranial fossa, contain cerebrospinal fluid and show no fat content [51].

\section{Lacrimal gland tumours}

Lacrimal gland tumours are classified as epithelial and non-epithelial lesions. Lacrimal gland epithelial tumours are similar to salivary gland tumours and constitute 40 $50 \%$ of all lacrimal masses. Half of these are benign mixed tumours (BMT), while the other half are malignant masses. Adenoid cystic carcinoma (ACC) is the most common lacrimal gland malignancy, followed by carcinoma ex-pleomorphic adenoma, adenocarcinoma, and mucoepidermoid carcinoma. Lymphoma, inflammatory conditions, and non-carcinomatous metastases constitute the non-epithelial lesions.

\section{Benign mixed tumour (BMT)}

BMT or pleomorphic adenoma originates mainly from the orbital lobe of the lacrimal gland. It is seen in middleaged patients (40-50 years) without gender predilection. administration. The $\mathrm{ADC}$ values are very low in the anterior part of the lesion $\left(\mathrm{ADC}=0.1 \times 10^{-3} \mathrm{~mm}^{2} / \mathrm{s}\right)$ due to fat and they are very high in the posterior component $\left(\mathrm{ADC}=1.8 \times 10^{-3} \mathrm{~mm}^{2} / \mathrm{s}\right.$ ) due to fluid. f. DTI $3 \mathrm{D}$ tractography reconstruction of the optic nerves (green) viewed from above and from the left. Right optic nerve fibres (white thin arrows) and left optic nerve fibres (green thin arrows) are normal and have similar FA and ADC values. Thick arrow points to the dermoid
Clinical signs include a painless, slow-growing mass in the lateral orbit, usually persisting for more than 12 months. Proptosis may be seen. Although benign, these tumours can show recurrence and malignant transformation (carcinoma ex-pleomorphic adenoma), primarily in cases where only a biopsy or incomplete excision were performed. Hence, meticulous surgical excision and careful pathological examination for capsular invasion is mandatory $[53,54]$.

BMT arise from the ductal system of the gland. These encapsulated tumours contain interspersed epithelial and stromal components. Necrosis, hyalinization, myxoid, and mucinous degeneration may be seen [26, 53, 54]. Pseudopodia and satellite tumours have been described histologically in more than $50 \%$ of BMT arising in the parotid gland, these histological features being associated with a higher risk of recurrence [55]. Pseudopodia, satellite lesions, and bony remodelling can equally be seen in BMTs arising in the lacrimal glands; they should not be misinterpreted as a feature of malignancy (Fig. 9). On CT, BMT are seen as well-circumscribed, round-oval masses with varying attenuation depending upon their composition and cellularity. Highly cellular masses appear homogeneous. Cystic degeneration may give rise to 

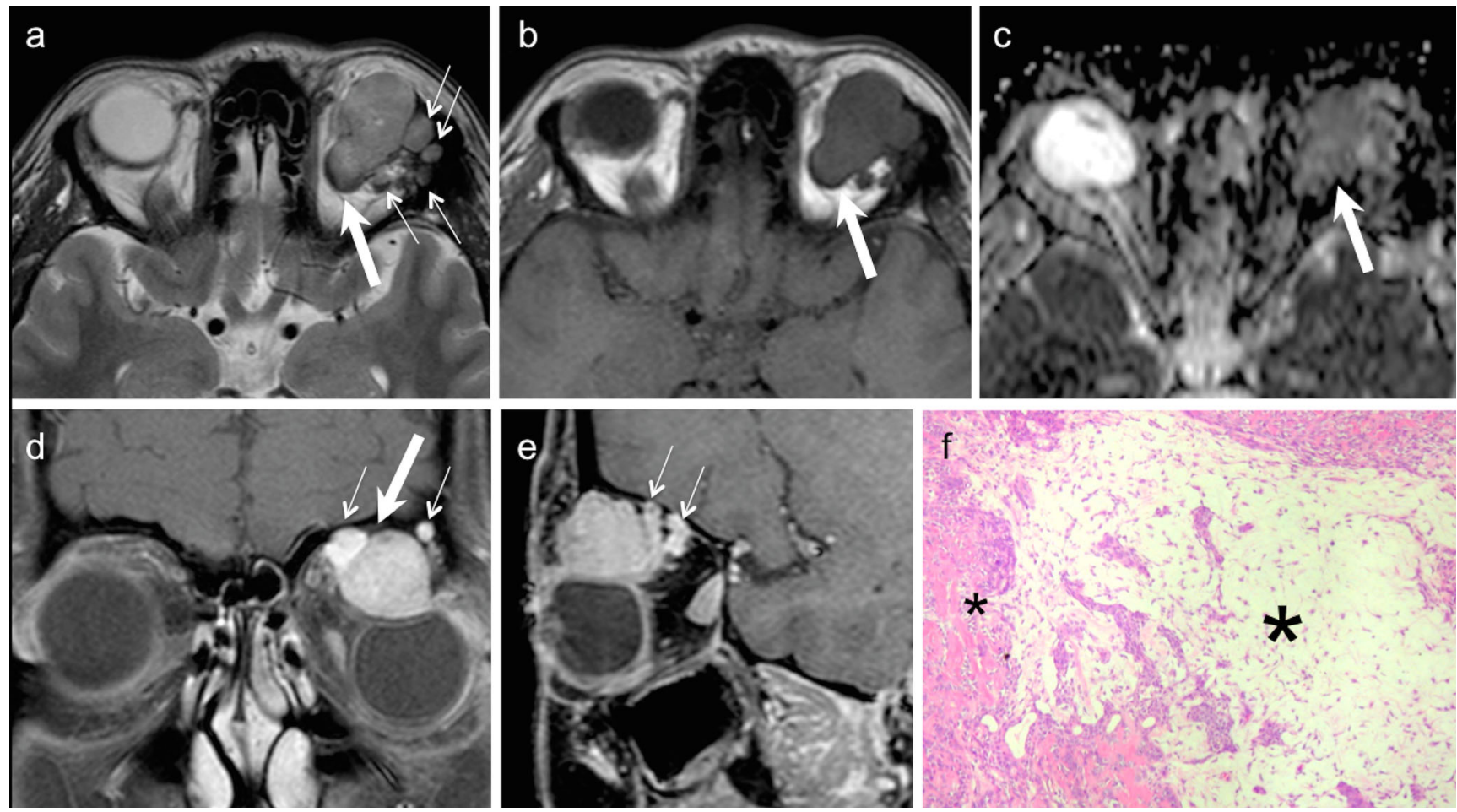

Fig. 9 42-year-old female patient with BMT of the left lacrimal gland. a. Axial T2W MR image shows a well-circumscribed polypoid mass (thick arrow) of moderately hypointense signal involving the left lacrimal gland. Note small satellite nodules (thin arrows). b. Corresponding T1W image shows non-specific lesion hypointensity (arrow). c. ADC map reveals increased diffusion $\left(\mathrm{ADC}=1.6 \times 10^{-3} \mathrm{~mm}^{2} / \mathrm{s}\right)$. d. Coronal contrastenhanced FS T1W MR image of the same patient demonstrates strong enhancement within the mass (thick arrow) and within the peripheral nodules (thin arrows). e. Sagittal reformatted $0.6 \mathrm{~mm}$ thin image from

contrast-enhanced 3D VIBE better illustrates scalloping of the orbital roof by the peripheral "grape-like" nodules (thin arrows). e. Photomicrograph of the surgical specimen (original magnification 100x, H\&E stain) illustrates the characteristic histological features of pleomorphic adenoma with medium sized cells with an eosinophilic cytoplasm and myoepithelial cells (small asterisk) partly surrounded by a myxoid matrix (large asterisk). The peripheral nodules seen on MRI corresponded histologically to pseudopodia and satellite nodules. Bony invasion by pseudopodia was confirmed histologically

hypodense/inhomogeneous appearance. Lacrimal fossa deformation and calcifications may be seen on bonewindow CT. On MRI, a heterogeneous signal is identified, especially on $\mathrm{T} 2 \mathrm{~W}$ images with moderate/heterogeneous or homogenous contrast enhancement (Fig. 9). Infiltration of the adjacent orbital tissue and poorly defined margins suggest malignant transformation [53, 54]. Motoori et al proposed that the detection of myxomatous tissue on $\mathrm{T} 2 \mathrm{~W}$, inversion recovery, DWI, and dynamic contrastenhanced sequences help to differentiate BMT from other malignant tumours [56].

Similar to salivary gland BMT, lacrimal gland BMT do not show restricted diffusion (Fig. 9) [56-58]. Sepahdari et al have reported a single BMT of the lacrimal gland with ADC value of $1.37 \times 10^{-3} \mathrm{~mm}{ }^{2} / \mathrm{s}$ [9], Razek et al have reported ADC ranges in BMT between 1.62-1.76 $\times 10^{-3} \mathrm{~mm}^{2} / \mathrm{s}$ [12], whereas Elkhamary et al have reported ADC ranges between 1.18$1.23 \times 10^{-3} \mathrm{~mm}^{2} / \mathrm{s}$ [58]. These ADC values are significantly higher than the malignancy threshold value of $1.0 \times 10^{-3} \mathrm{~mm}^{2} /$ s $[9,10,12,56-58]$. Thus, ADC values appear to be highly sensitive and specific in differentiating BMT from malignant tumours of the lacrimal gland.

\section{Malignant epithelial lacrimal gland tumours}

Approximately $50 \%$ of epithelial lacrimal gland tumours are malignant. Malignant tumours of epithelial origin include adenoid cystic carcinoma (ACC), mucoepidermoid carcinoma, adenocarcinoma, squamous cell carcinoma, and undifferentiated carcinoma types, such as the mammary analog secretory carcinoma of salivary origin (Fig. 10). Overall, these tumours are quite rare, accounting for less than $5 \%$ of all lacrimal gland lesions. Nevertheless, malignant lacrimal gland tumours need to be recognized at an early stage, as they tend to have a high morbidity and mortality. ACC is a high-grade malignancy accounting for $29 \%$ of all epithelial lacrimal gland tumours and as many as $50 \%$ of all malignant epithelial lacrimal gland neoplasms. Its incidence peaks in the 4 th decade. Patients present with a hard mass in the upper lateral orbit, often with pain caused by perineural spread or bony invasion. Perineural spread indicates poorer prognosis. Radical resection with wide margins is performed for small, low-grade tumours. Survival rates vary from 5 years in $40 \%$ of patients to 15 years in $58 \%$ of patients $[1,2,53,54]$. Histologically, these tumours are 

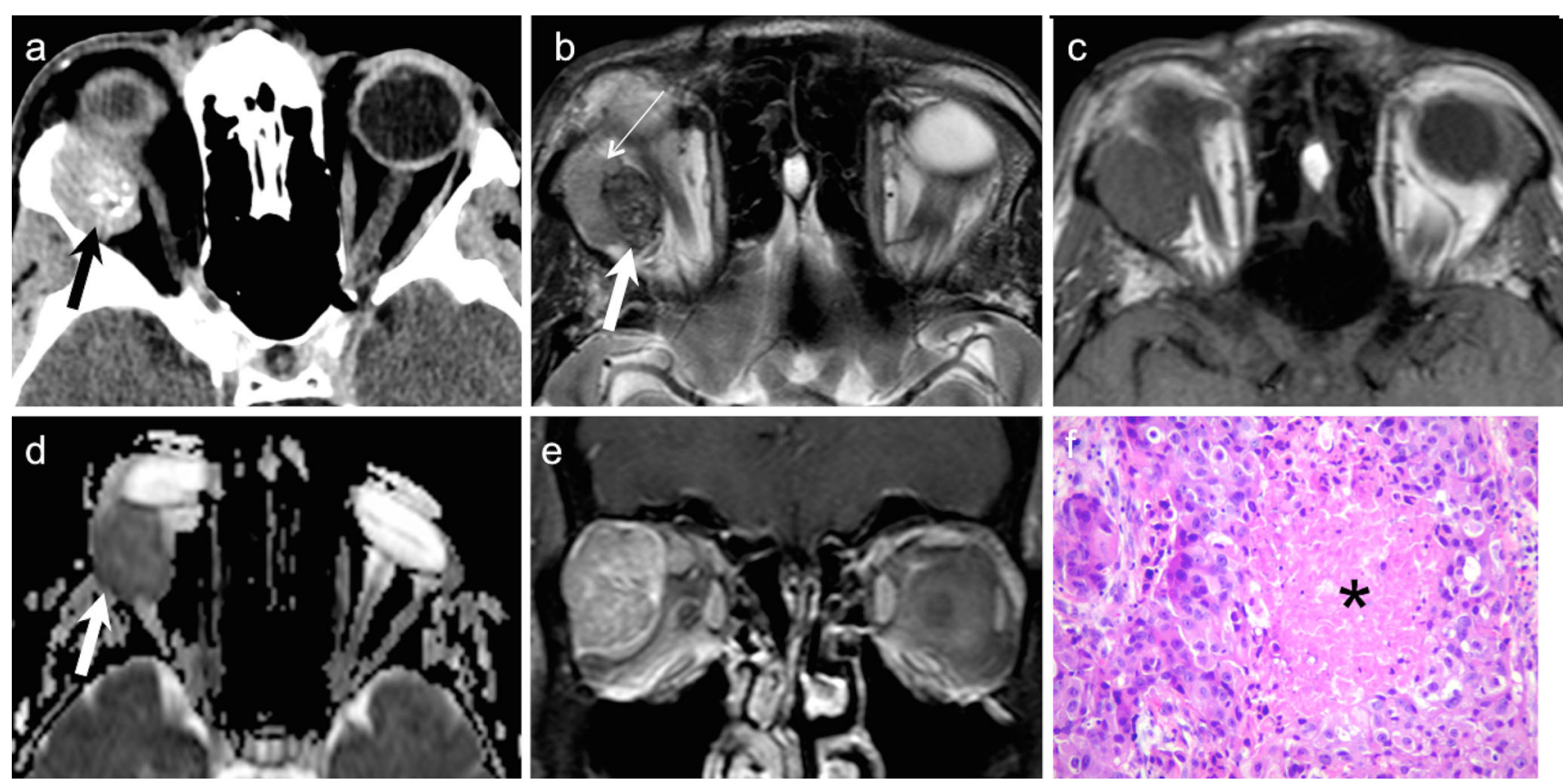

Fig. 10 65-year-old male patient with undifferentiated ductal carcinoma of the right lacrimal gland (mammary analog secretory type). a. Axial NECT of the orbits shows a well-circumscribed, hyperdense mass with stippled calcifications involving the right lacrimal gland. The calcifications were misdiagnosed as phleboliths, which led to the initial diagnosis of a cavernous hemangioma. b. Axial T2W MR image of the same patient shows that the lacrimal gland mass has a very hypointense posterior component (thick arrow) and an anterior moderately hypointense portion (thin arrow). c. Corresponding axial T1W MR

characterized by absence of a mesenchymal matrix. They show different histological types like tubular, comedo-carcinoma, basaloid, or cribriform patterns. The cribriform pattern consists of sheets of basaloid epithelial cells and surrounding spaces of varying shapes giving rise to a characteristic Swiss cheese appearance $[26,53,54]$.

CT shows non-specific findings; often a well or poorly circumscribed mass involving the lacrimal gland with associated bony destruction in $70 \%$ cases (Fig. 11). Low-grade tumours may exactly mimic BMT with a well-circumscribed margin and no bony destruction. Intratumoural calcifications are more common in ACC, in adenocarcinoma and in undifferentiated epithelial tumours (Fig. 10) than in a BMT and should not be misinterpreted as phleboliths. ACC often appears hypointense on T1W images, hypo- or hyperintense on $\mathrm{T} 2 \mathrm{~W}$ images and shows prominent post-contrast enhancement (Fig. 11). Fat-saturated CEMRI is ideal for local tumour staging and for evaluating perineural spread $[1,2,53,54]$. In general, most ACC will show perineural spread at microscopic examination; however, radiologically, perineural spread may remain undetected unless involvement of major nerve trunks, such as where the supraorbital nerve occurs (Fig. 11).

Elkhamary SM et al have reported $0.8 \times 10^{-3} \mathrm{~mm}^{2} / \mathrm{s}$ as the mean ADC value for ACC (which is significantly lower than BMT and other benign lesions), but significantly higher than image shows that the mass is isointense to the rectus muscles. d. ADC map reveals restricted diffusion $\left(\mathrm{ADC}=0.9 \times 10^{-3} \mathrm{~mm}^{2} / \mathrm{s}\right.$ ), suggesting a malignant tumour. e. Coronal FS contrast-enhanced T1 W image shows moderate tumour enhancement and lobular appearance. f. Photomicrograph (original magnification 100x, H\&E stain) shows a highly cellular tumour with atypical nuclei and mitoses and areas of necrosis (asterisk). There were numerous areas of microscopic perineural spread and lymphatic invasion not detected by imaging

lymphomas $\left(0.6 \times 10^{-3} \mathrm{~mm}^{2} / \mathrm{s}\right)$ [58]. ADC values can help to differentiate between carcinomas, lymphomas, metastases, and inflammatory disorders (described in subsequent sections) $[1,9-12,53,54,58]$. Nevertheless, ACC is often indistinguishable from other malignant epithelial tumours of the lacrimal gland on MRI with DWI (Fig. 10) unless perineural spread, which is more common in ACC, is detected (Fig. 11). Occasionally, mucoepidermoid carcinomas may show high T1 signal due to their mucin content.

ACC is commonly hypermetabolic on PET CT. Baek et al have reported that PET CT was successful in detecting both the primary lesion and distant metastases of lacrimal gland ACC [17-19, 59].

\section{Rhabdomyosarcoma (RMS)}

RMS is the most common malignant mesenchymal tumour of childhood. The orbit is the most common location in the headneck with $40 \%$ of tumours appearing there. The incidence peaks between 5-10 years of age, as shown by positron emission tomography, with a slight predilection for boys. RMS typically arises in the extraconal compartment; however, intraconal extension is known. It presents with rapidly progressive proptosis, ptosis, or signs of inflammation often prompting urgent imaging. It is an aggressive tumour and 

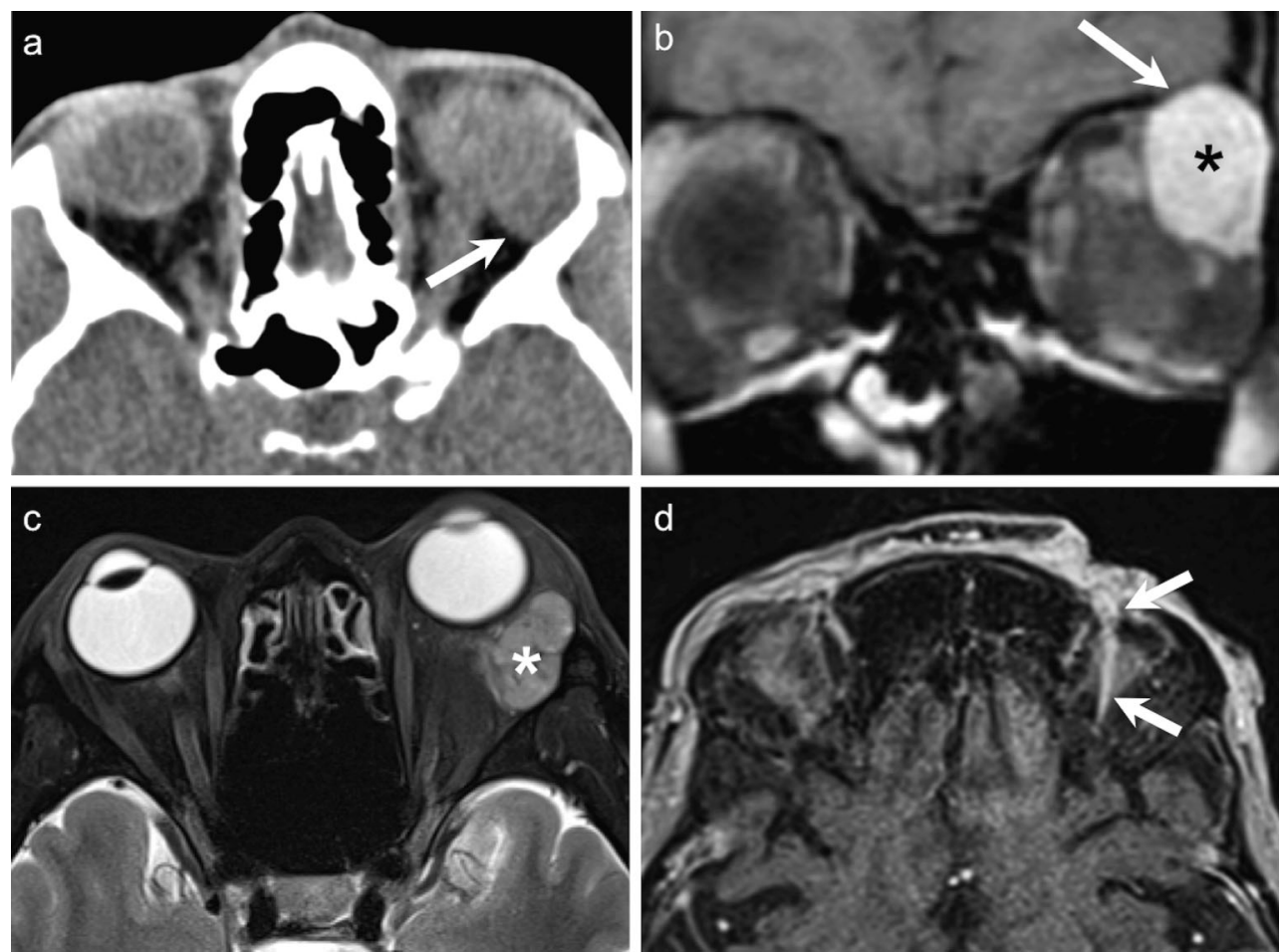

Fig. 11 50-year-old male patient with histologically proven ACC of the left lacrimal gland. Axial NECT of the orbits shows a well-circumscribed, slightly hyperdense mass (arrow) involving the left lacrimal gland. There is suggestion of minimal adjacent bony scalloping. b. Coronal contrastenhanced FS TIW image of the same patient shows avid, homogeneous, contrast enhancement within the mass (asterisk), mimicking a cavernous hemangioma. There is thinning of the overlying left frontal bone (arrow). c. Axial T2W image of the same patient showing intermediate signal

commonly infiltrates into the adjacent sinuses, orbital fissures, cavernous sinus, and middle cranial fossa. Hematogenous spread can occur to the lungs. Surgery and chemotherapy used in combination may achieve good survival rates. RT is avoided because of the potential risk of cataract, encephalopathy, and radiation-induced sarcomas [1, 2, 4, 26, 60].

RMS is believed to arise from primitive mesenchymal cells. RMS can be divided histopathologically into embryonal, pleomorphic, alveolar, and botryoid subtypes. The alveolar subtype is the most anaplastic variant characterized by tumour cells spreading along soft-tissue septa. The rhabdomyoblast is the diagnostic cell in all types which shows granular eosinophilic cytoplasm with thick and thin filaments. Conventional H\&E staining is complimented with immunohistochemistry to detect myoglobin and desmin which support the diagnosis [26, 60].

$\mathrm{CT}$ and MR are often used in combination to assess tumour size, extraorbital extension, bony destruction and intracranial involvement. RMS appears isodense to muscle on NECT and usually shows significant enhancement on CECT. It appears iso-intense to muscle on T1W images, hypo or hyperintense on $\mathrm{T} 2 \mathrm{~W}$ images and shows marked enhancement on CEMRI (Fig. 12). Necrosis and calcification is uncommon $[1,2,4,26,60]$.

within the mass (asterisk) suggesting high cellularity, a feature that is uncommon in hemangioma. The patient underwent surgery, which revealed ACC with multiple areas of microscopic perineural spread not detected by imaging. $\mathbf{d}$. Contrast-enhanced axial T1W image obtained in a different patient 10 years after surgery of an ACC of the left lacrimal gland shows macroscopic perineural tumour recurrence along the supraorbital nerve (arrows). The findings were confirmed histologically

Many benign and malignant entities may mimic the imaging features of RMS; however, the presence of unilateral, rapidly progressive proptosis in a child must always raise concern for RMS. Orbital cellulitis may show similar clinical features. On imaging, both conditions may show an orbital mass with adjacent paranasal sinus involvement. Fever, leukocytosis, and orbital fat stranding/abscess formation suggest infection. Although capillary hemangiomas manifest in a younger agegroup (12-18 months), they may mimic a very vascular RMS. Capillary hemangiomas often show multiple flow-voids, avid contrast enhancement, and high flow on time-resolved MR angiography. Associated cutaneous hemangiomas are seen in one third of cases. In cases with equivocal clinical and imaging findings, a biopsy is necessary to reach the diagnosis. Langerhans cell histiocytosis may present with orbital involvement in $23 \%$ cases. It can appear as an aggressive soft tissue mass with bony invasion, similar to RMS. It may be associated with diabetes insipidus. Again, a biopsy may be necessary to differentiate between the two. Neuroblastoma metastases to the orbit can mimic RMS on imaging. The finding of a primary tumour in the retroperitoneum or posterior mediastinum helps to arrive at the correct diagnosis [60]. Lymphoma is rare in children accounting for less than 5- 
Fig. 12 4-year-old girl with a left orbital RMS. a. Axial T2W MR image of the orbits shows a conalextraconal polypoid mass of moderately low signal intensity located superior to the left globe. b. ADC map shows restricted diffusion $\left(\mathrm{ADC}=1 \times 10^{-3} \mathrm{~mm}^{2} /\right.$ s), suggestive of malignancy. c. Coronal contrast-enhanced FS T1W MR image shows avid enhancement within the lesion. d. Axial FDG PET/CT image of the same patient shows high SUVs $($ SUVmean $=6$, SUVmax $=9$ ). Although the lesion may mimic a cavernous hemangioma on the $\mathrm{T} 1 \mathrm{~W}$ and $\mathrm{T} 2 \mathrm{~W}$ images, the low ADC and the high FDG uptake strongly suggest a malignant tumour. Histology revealed embryonal rhabdomyosarcoma
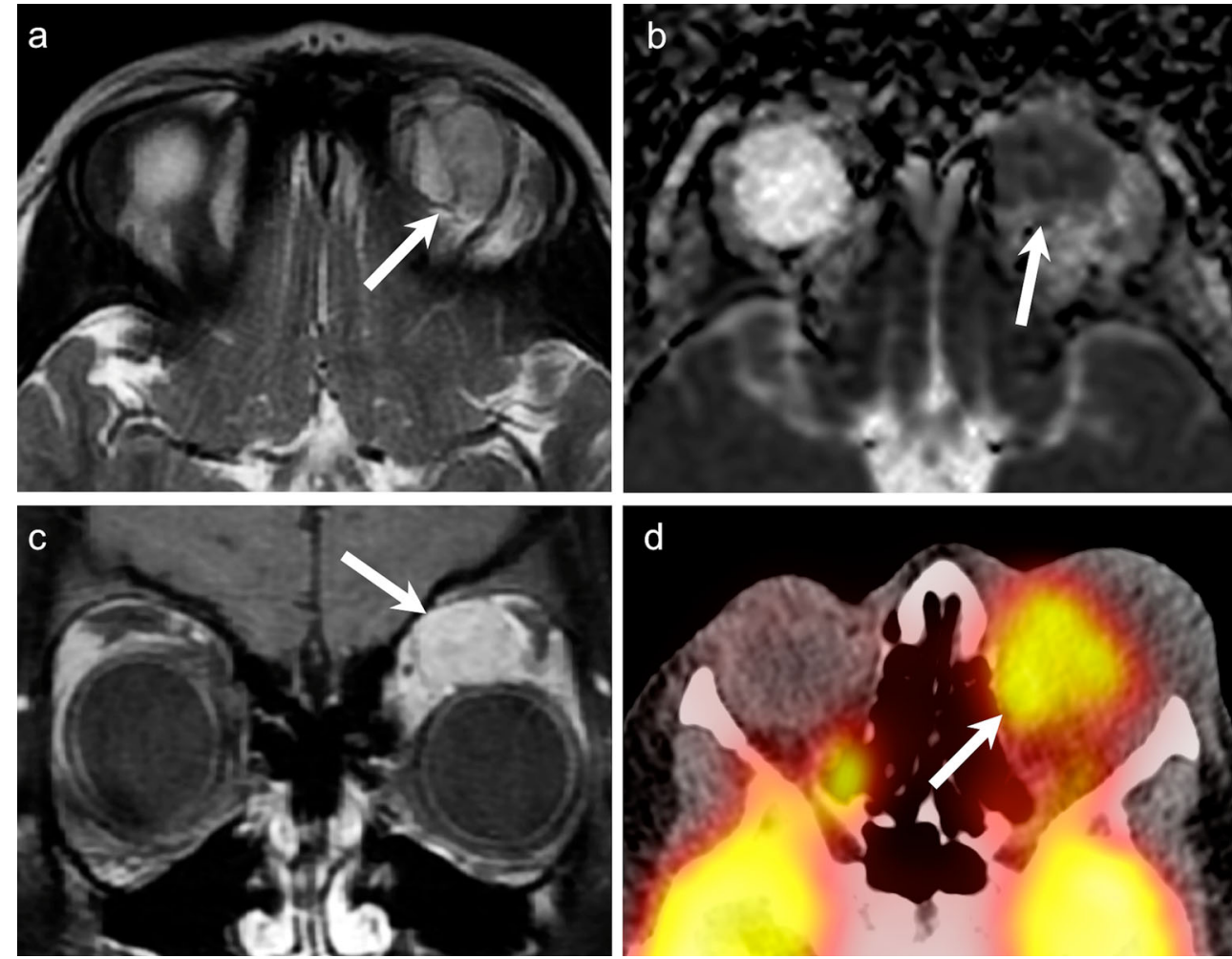

$10 \%$ of orbital lesions. Unlike RMS, it commonly involves the lacrimal gland, appears hypointense on T2W images, encases rather than distorts the globe and shows even lower ADC values than RMS $[4,13,60]$.

Sepahdari et al have reported $0.72 \times 10^{-3} \mathrm{~mm}^{2} / \mathrm{s}$ as the mean ADC value in a cohort of 12 cases of orbital RMS [10] (Fig. 12). Lope et al have stated that the low ADC values of RMS can help to differentiate them from haemangiomas which usually show high ADC values [13]. Hassold et al have reported a case of orbital RMS where low ADC value within the recurrent tumour $\left(0.62 \times 10^{-3} \mathrm{~mm}^{2} / \mathrm{s}\right)$ helped to differentiate it from post-therapeutic changes [61].

PET CT is a valuable adjunct for the grading and staging of paediatric sarcomas [62]. FDG uptake is known to correlate with the tumour grade of mesenchymal sarcomas $[62,63]$ (Fig. 12). PET CT has been deemed superior at detecting osseous and nodal metastases of RMS than conventional imaging $[62,64]$. Whole-body MRI has shown comparable results as PET CT, and more accuracy as compared to skeletal scintigraphy in the detection of osseous metastases of RMS [65]. MRI PET may potentially improve the staging, followup and post-treatment assessment of RMS [22].

\section{Lymphoma}

Lymphoma of the ocular adnexa is the most common orbital malignancy accounting for $55 \%$ of adult malignant orbital tumours. This is a heterogeneous group of tumours composing approximately 1-2 \% of non-Hodgkin's lymphomas (NHL) and $8 \%$ of extranodal mucosa-associated lymphoid tissue (MALT) lymphomas. Highest incidence is seen in the 6th7th decade. Lymphoma can be primary to the orbit or secondary to systemic disease. Secondary ocular involvement occurs in $2-5 \%$ of patients with advanced systemic NHL. About $75 \%$ of patients with primary orbital lymphoma eventually develop systemic disease. Patients with Sjögren's syndrome have a higher risk of developing extranodal NHL as compared to age-, race-, and sex-matched controls. Sjögren's syndrome, either primary or secondary (due to rheumatoid arthritis, systemic lupus erythematosus, or progressive systemic sclerosis), is an autoimmune disease of the exocrine glands characterized by lymphocytic infiltration of the affected glands. About $6 \%$ of patients with Sjögren's syndrome show clinically evident NHL manifestations. Most NHL seen in patients with Sjögren's syndrome are of B cell lineage and can occur in the salivary glands, lacrimal glands, lymph nodes, lung, and thyroid. In the orbit, NHL can be unilateral or bilateral. In the orbit, the anterior extraconal space and lacrimal gland are most commonly involved. Involvement of the lacrimal gland is seen in about $7 \%$ of all NHL cases associated with Sjögren's syndrome and manifests clinically with lacrimal gland enlargement. Other clinical manifestations of orbital lymphoma include painless proptosis and motility disturbances. Wholebody staging is necessary when orbital lymphoma is 

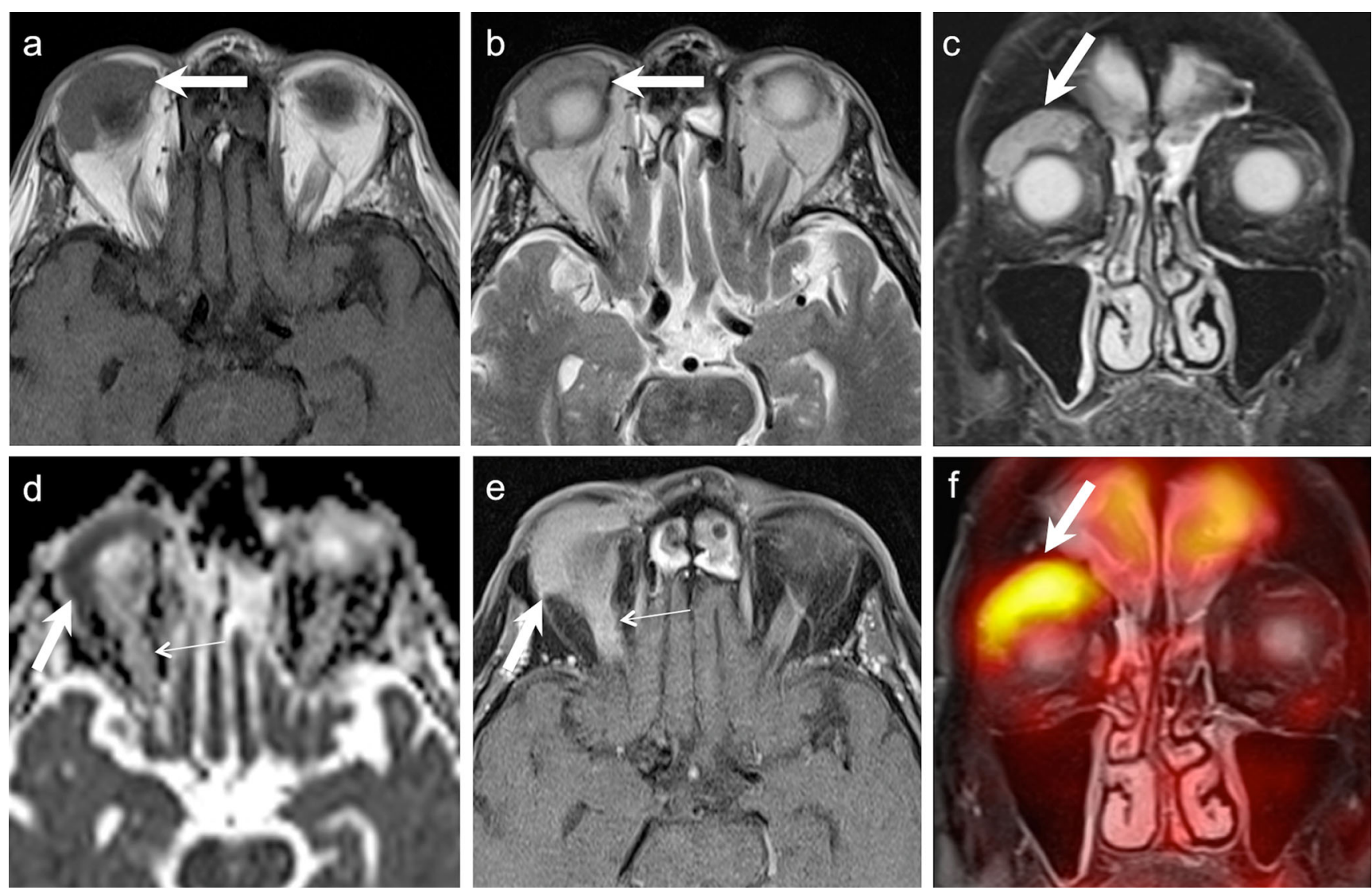

Fig. 13 80-year-old male patient with lymphoma of the right orbit. a. Axial T1W image of the orbits shows a hypointense, well-demarcated anterior conal-extraconal lesion (arrow). Axial T2W (b) and coronal STIR (c) images demonstrate intermediate signal intensity of the bulky mass (arrows). Note homogenous aspect. ADC map (d) reveals restricted diffusion (arrow) with very low $\mathrm{ADC}$ values $\left(\mathrm{ADC}=0.6 \times 10^{-3} \mathrm{~mm}^{2} / \mathrm{s}\right)$ characteristic of lymphoma. Axial contrast enhanced T1W image (e)

shows homogenous enhancement of the mass (arrow). There is enhancement of the superior rectus muscle (thin arrow) suggesting possible involvement. However, the ADC map clearly shows that the superior rectus muscle has no restricted diffusion (thin arrows in $\mathrm{d}$ and e). f. Coronal fused PET and STIR image from FDG PET/MRI reveals high tracer uptake within the mass (SUVmean $=4.8$, SUVmax $=6.7$ ). No other lymphoma manifestations were detected in the body

diagnosed and is most often done with FDG PET CT. Lowgrade tumours respond well to RT while chemotherapy is necessary for high-grade or systemic disease [1, 4, 18, 66, 67].

Histologically, ocular adnexal MALT lymphomas are characterized by a heterogeneous cell population, consisting of monocytoid, plasmacytoid, and centrocyte-like cells, with occasional blasts in the marginal zone surrounding lymphoid follicles. Pathognomonic histological features include "follicular colonization" and the formation of "lymphoepithelial lesions" through the invasion of neighbouring epithelial structures by nests of MALT lymphoma cells. Immunophenotypically, orbital MALT lymphomas show dense CD $20^{+}$, CD $10^{-}$and CD $23^{-}$B-cell lymphocytic infiltrates which helps to differentiate them from benign lymphoproliferative disorders and other small B-cell lymphomas. MALT lymphomas are typically negative for CD5, which helps to differentiate them from mantle-cell lymphomas [26, 67].

NECT typically shows a hyperdense mass involving the lacrimal gland. The tumour usually molds to encase surrounding orbital structures. Significant enhancement may be seen on CECT. Bony destruction or perineural spread suggests an aggressive histology. High-cellularity tumours appear moderately hypointense on T1W and T2W MR images (Figs. 13 and 14). CEMRI usually shows avid enhancement (Fig. 14). At times, isolated involvement of the extraocular muscles or diffuse ill-defined orbital infiltration may be seen (Fig. 14) [1, 4, 66, 67].

Other infiltrative $\mathrm{T} 2$ moderately hypointense lesions namely benign orbital lympoproliferative disorders (OLPD), inflammatory orbital pseudotumour (IOP), and granulomatous diseases such as sarcoidosis and metastases (described subsequently) are common imaging differentials $[1,9,68,69]$.

Orbital lymphomas typically show very low ADC values, usually between $0.44-0.92 \times 10^{-3} \mathrm{~mm}^{2} / \mathrm{s}$ (Figs. 13 and 14). Studies have proven that orbital lymphoma can be differentiated from IOP (ADC range $1.02-2.28 \times 10^{-3} \mathrm{~mm}^{2} / \mathrm{s}$ ) with $100 \%$ accuracy using an ADC threshold of $1.0 \times 10^{-3} \mathrm{~mm}^{2} / \mathrm{s}$ and an ADC ratio of less than $1.2 \times 10^{-3} \mathrm{~mm}^{2} / \mathrm{s}[9-12,68]$. Similarly, lymphomas show significantly lower ADC values 

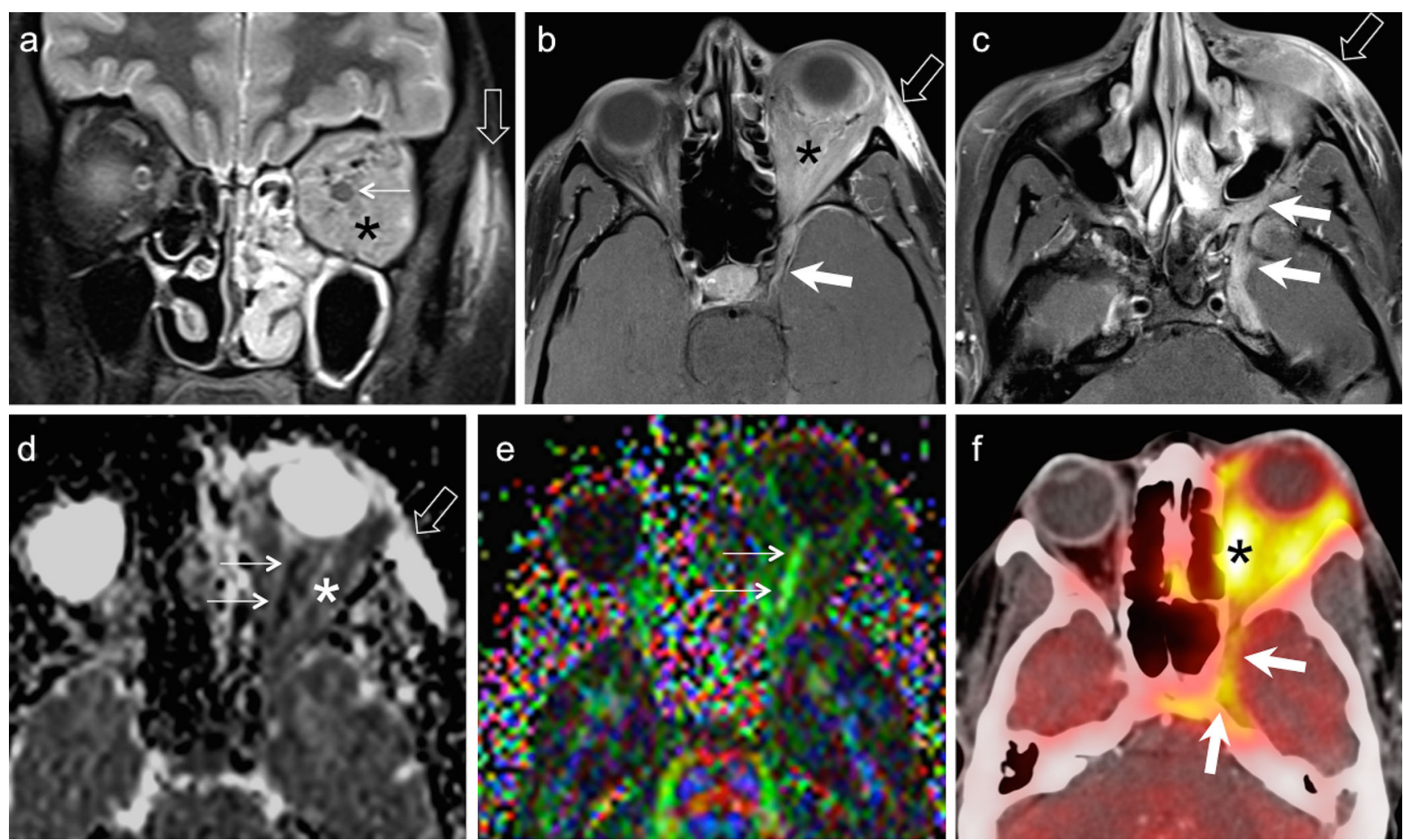

Fig. 14 35-year-old female patient with painful proptosis, loss of vision, and subcutaneous facial swelling. Biopsy of the face performed in an outside institution suggested inflammatory pseudotumour. a. Coronal STIR image shows an ill-defined, moderately hyperintense lesion involving the entire left orbit (asterisk) and encasing the optic nerve (thin arrow). The subcutaneous hyperintense, poorly defined area on the left (hollow arrow) corresponds to the biopsied region. b. Axial contrastenhanced FS T1W MR image of the same patient as in a. The left orbital lesion shows diffuse post-contrast enhancement (asterisk). Enhancing soft tissue is seen extending along the left superior orbital fissure into the left cavernous sinus (arrow) and the dura along the left greater wing of sphenoid. c. Axial contrast-enhanced FS T1W image at the level of the maxillary sinus demonstrates perineural spread along the pterygopalatine

as compared to Ig-G4 related disease (mean ADC $1.67 \times 10^{-3}$ $\mathrm{mm}^{2} / \mathrm{s}$ ) [69] as well as metastases (ADC range $0.9-1.6 \times 10^{-3}$ $\left.\mathrm{mm}^{2} / \mathrm{s}\right)[9,10,12]$. In a study by Haradome et al, the mean $\mathrm{ADC}$ and contrast-enhancement ratio of orbital lymphomas fossa and maxillary nerve (arrows). Hollow arrows in $\mathrm{b}$ and $\mathrm{c}$ point at extra-orbital involement. d. ADC map reveals restricted diffusion of the orbital lesion with very low $\mathrm{ADC}$ values $\left(\mathrm{ADC}=0.7 \times 10^{-3} \mathrm{~mm}^{2} / \mathrm{s}\right)$ suggesting lymphoma. The optic nerve shows even lower ADC values (thin arrows) due to compression and ischemia. e. Colour coded DTI map shows major reduction of FA values in the left optic nerve (thin arrows). FA values were $0.4-0.5$ on the left and $0.56-0.58$ on the right. f. FDG $\mathrm{PET} / \mathrm{CT}$ demonstrates high tracer uptake in the orbit (asterisk), along the superior orbital fissure and in the cavernous sinus and sphenoid (arrows) confirming findings revealed in $\mathrm{b}$. SUVmean $=10, \max =16$. Other hypermetabolic lesions were found in the neck nodes, mediastinum, and abdomen. Biopsy of orbital contents revealed NHL

was found to be significantly lower than that of benign OLPD, thereby aiding in their differential diagnosis [70].

Sarcoidosis may mimic lymphoma at clinical presentation and at cross-sectional imaging. Sarcoidosis is a chronic,
Fig. 15 Two different patients with FD involving the facial and orbital bones. Characteristic ground-glass appearance (asterisks) and expansion of the medullary cavity is seen in both cases on NECT. In $\mathbf{b}$, bony expansion of the right sphenoid wing and of the right anterior clinoid process causes severe narrowing of the right optic canal (arrow), thereby requiring decompressive surgery
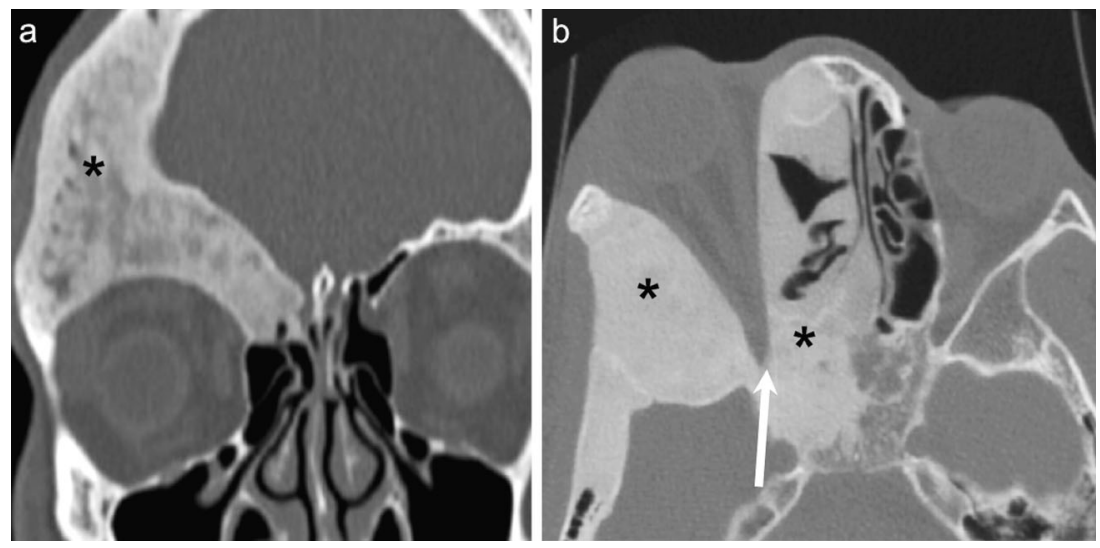

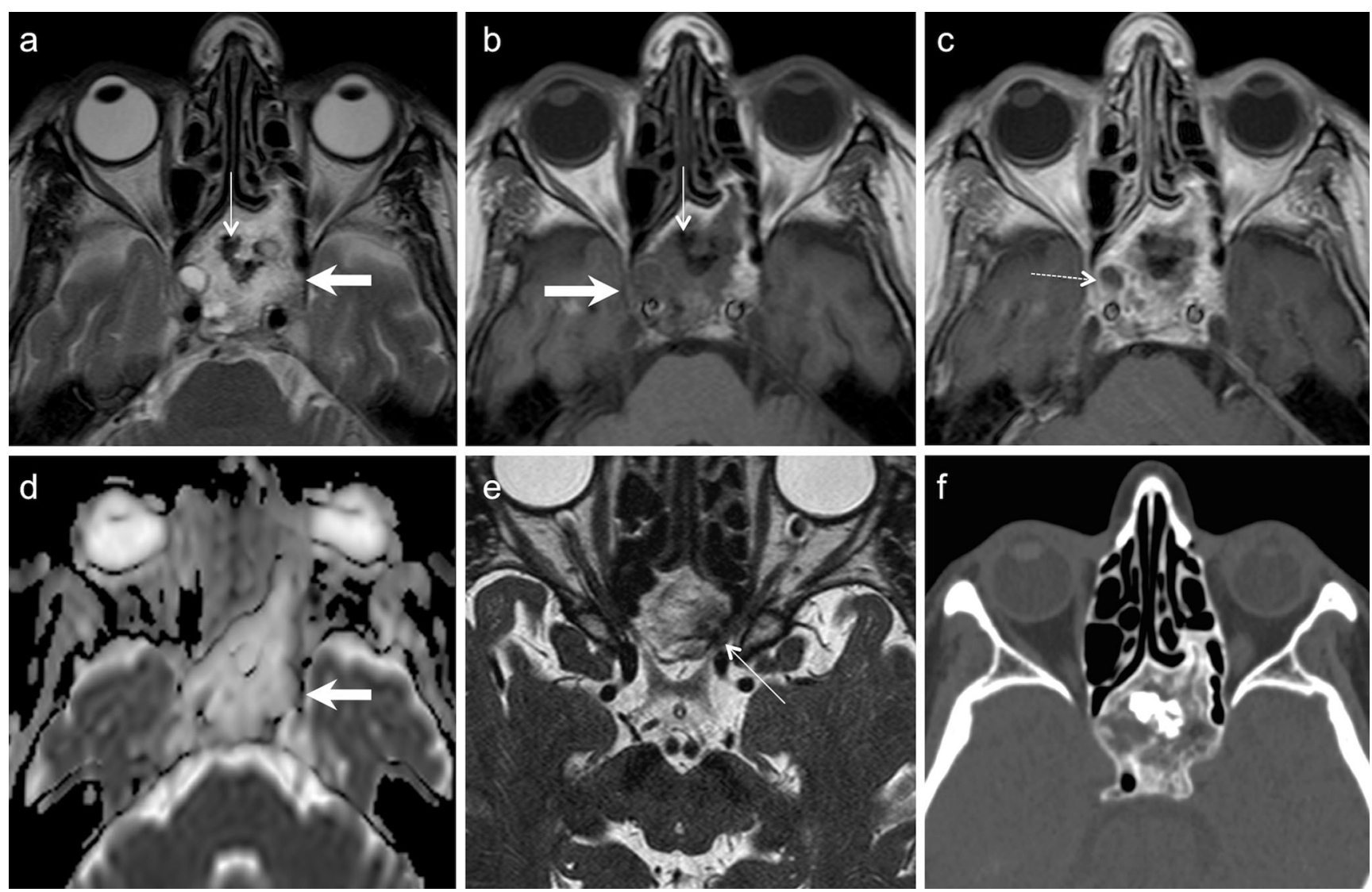

Fig. 16 64-year-old female patient with headache and left vision loss underwent MRI. a. Axial T2W image of the orbits shows deformity and expansion of the sphenoid body. The medullary cavity is replaced by tissue with mixed hyperintense (thick arrow)-hypointense (thin arrow) signal. b. Axial T1W image of the same patient shows the lesion to be mainly isointense to brain parenchyma (thick arrow) and with strongly hypointense central areas (thin arrow). On the left, there are hyperintense peripheral regions. c. Axial contrast-enhanced T1W image shows inhomogeneous contrast enhancement (thick arrow) and cystic portions

multisystemic, granulomatous disorder which commonly involves the orbit. It can cause mass-like lacrimal gland and muscle infiltration (often bilateral), optic nerve thickening

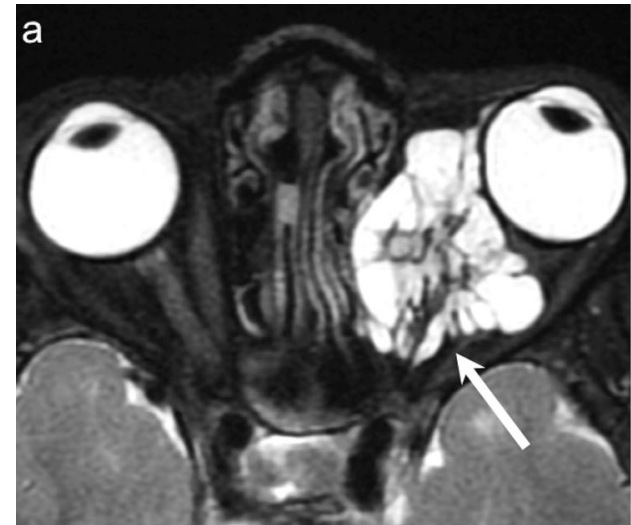

Fig. 17 15-year-old girl with a left orbital VLM. a. Axial FS T2W image shows a mutliloculated cystic lesion involving the intraconal and extraconal compartments of the left orbit (arrow). The strongly (dashed arrow). d. $\mathrm{ADC}$ map reveals increased diffusion $(\mathrm{ADC}=2.3 \times 10$ ${ }^{-3} \mathrm{~mm}^{2} / \mathrm{s}$ ) suggesting a benign lesion (thick arrow). FD was suspected. e. Oblique reformatted axial image from 3D T2W sequence shows compression of the left optic nerve (thin arrow) at the level of the optic canal. f. Axial NECT image shows ground-glass appearance and irregular ossification of the involved bones. Part of the lesion was resected to decompress the right optic nerve. Histology revealed psammomatous variant of FD

and enhancement or pseudotumoural intra-orbital masses. There may be associated intracranial extension. The imaging findings may be exactly similar to lymphoma, even on DWI

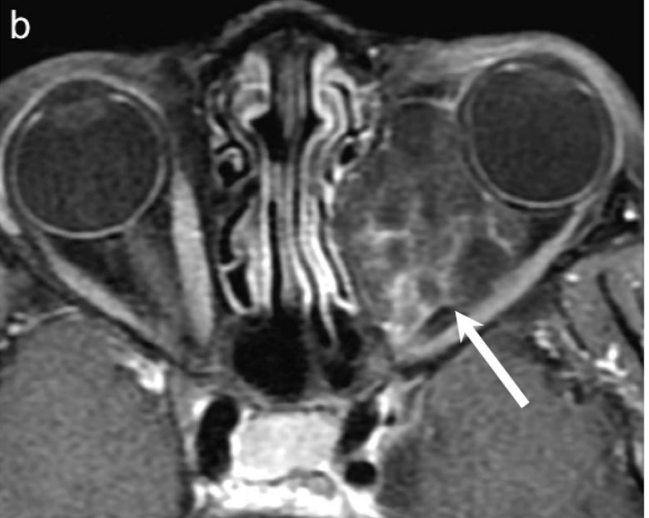

hypointense septae of the VLM show hemosiderin staining. b. Contrastenhanced FS T1W MR image of the same patient shows minimal enhancement (arrow) along the intervening septae of the VLM 

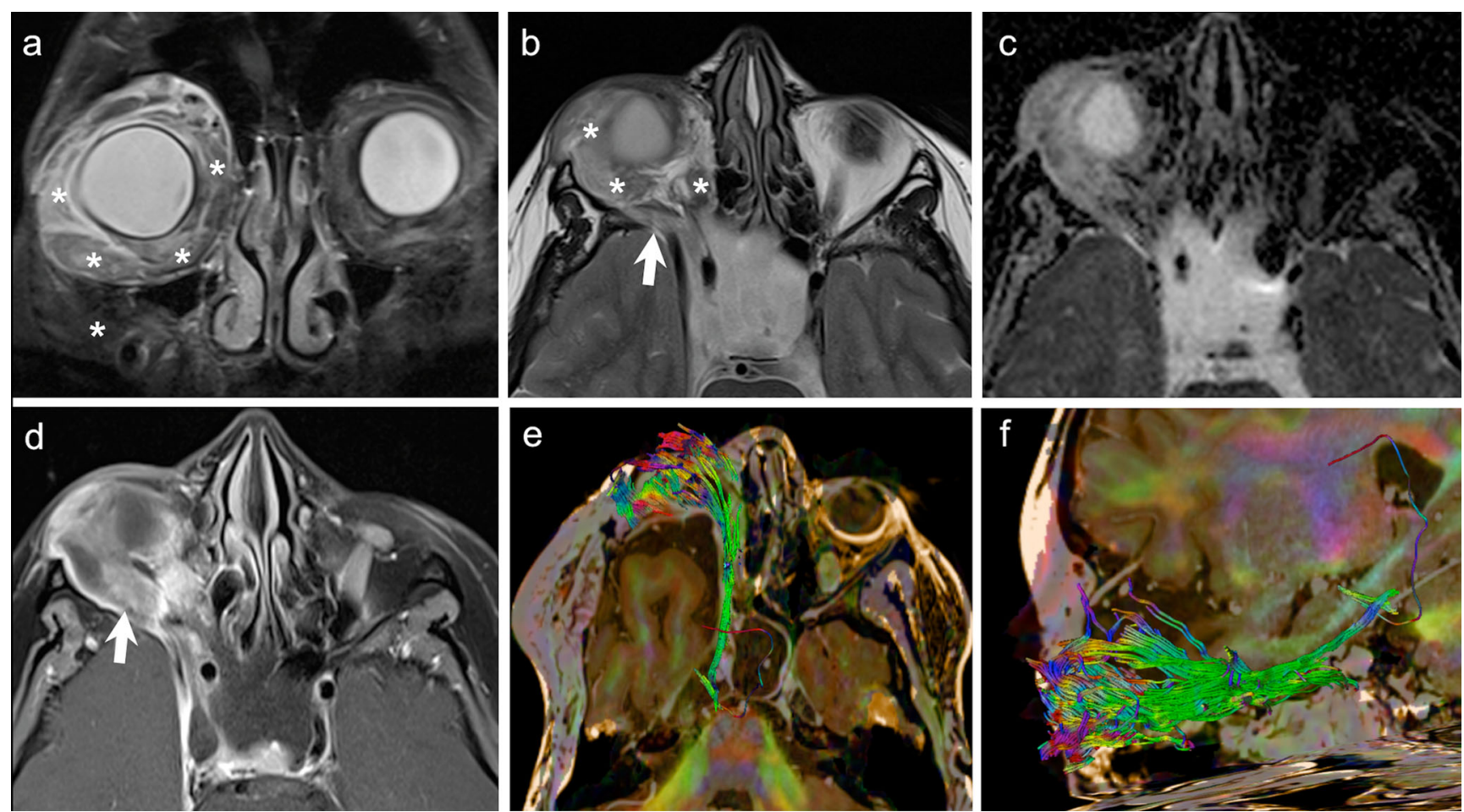

Fig. 18 28-year-old female patient with NF-1. MRI was performed for pre-surgical planning. Coronal STIR (a) and axial T2W (b) images show poorly circumscribed, serpentine masses (asterisks) within the right orbital conal-extraconal and preseptal compartments. Findings are typical of an OPNF. There is associated dural ectasia of the right optic nerve sheath. Classic right-sided sphenoid wing dysplasia is also noted

[12]. Bilateral hilar adenopathy, lung lesions, and elevated serum angiotensin-converting enzyme levels point to sarcoidosis. Often, a biopsy is required to demonstrate the noncaseating granulomas of sarcoidosis.

Lymphomas commonly show high FDG uptake (Figs. 13 and 14). Whole body PET CT has become part of standard staging of orbital lymphomas and is probably used more widely for this disease than any other orbital tumour Although, low-grade MALT lymphomas may show relatively low FDG uptake, PET CT has shown very good results in the detection of systemic metastases of even low-grade orbital lymphomas, which were not detected by conventional imaging [18, 20, 71, 72]. MRI PET holds promise in evaluating post-treatment response of lymphomas, especially in children $[22,73]$.

\section{Bone and sinus compartment}

\section{Fibrous dysplasia (FD)}

FD is a developmental disorder of the bone in which normal bone marrow is replaced by fibro-osseous tissue with expansion of the medullary cavity. Cranio-facial fibrous dysplasia is commonly involves the frontal, ethmoid or sphenoid bones. It is typically seen in children and adolescents with slight female predilection. FD is monoostotic in $70-80 \%$ cases and (arrow). The ADC map (c) shows no restriction of diffusion $(\mathrm{ADC}=1.3 \times$ $10^{-3} \mathrm{~mm}^{2} / \mathrm{s}$ ) in keeping with the benign histology. d. Axial contrastenhanced FS T1W image shows heterogeneous strong contrast enhancement of the OPNF (arrow). Axial (e) and sagittal (f) DTI 3D tractography views show complete disorganization of fibres within the OPNF surrounding the globe and optic nerve

polyostotic in the rest. Orbital bony involvement leads to hypertelorism, exophthalmos, visual impairment and blindness. Surgery is needed to correct facial deformity or severe optic nerve compression [1, 26, 51].

FD shows replacement of lamellar bone with abnormal metaplastic immature woven bone, forming irregular curvilinear trabeculae on histology; this appearance has been described as "Chinese characters" or alphabet soup. The fibrous portion contains bland spindle cells without significant mitotic activity. Haemorrhage and cystic changes may be seen [26, 51].

Radiographs and CT shows bony expansion with "ground glass" appearance (Fig. 15). There may be consequent narrowing of the optic nerve canal and impingement of the optic nerve (Figs. 15 and 16). The fibrous stroma and osteoid material typically appears hypointense on $\mathrm{T} 1 \mathrm{~W}$ images (Fig. 16). On T2W images the signal intensity may be low (18-38\% cases), intermediate (18\%) or high (62-64\%) (Fig. 16). Marked enhancement may be seen on CEMRI [1, 51] (Fig. 16), as well as fluid-fluid levels. Similar to benign lesions elsewhere in the head and neck, FD shows significantly higher ADC values compared to malignant bone tumours [74, 75] (Fig. 16).

FD can appear hypermetabolic on PET CT with high SUV values thereby simulating malignancy $[76,77]$. Nevertheless, 
Fig. 19 35-year-old female patient with left histologically proven orbital IOP. a. Axial T2W image shows a plaque-like hypointense extraconal lesion (arrowhead), adjacent to the lateral rectus muscle. Lymphoma was considered as an imaging differential. b. ADC map of the orbits of the same patient shows an $\mathrm{ADC}$ value of $1.1 \times 10^{-3} \mathrm{~mm}^{2} / \mathrm{s}$ (arrowhead), which is higher than the ADC value expected for lymphoma. c. Axial PET/CT image of the same patient shows high FDG uptake within the lesion (arrow) (SUVmean $=5$, SUVmax =6) mimicking lymphoma
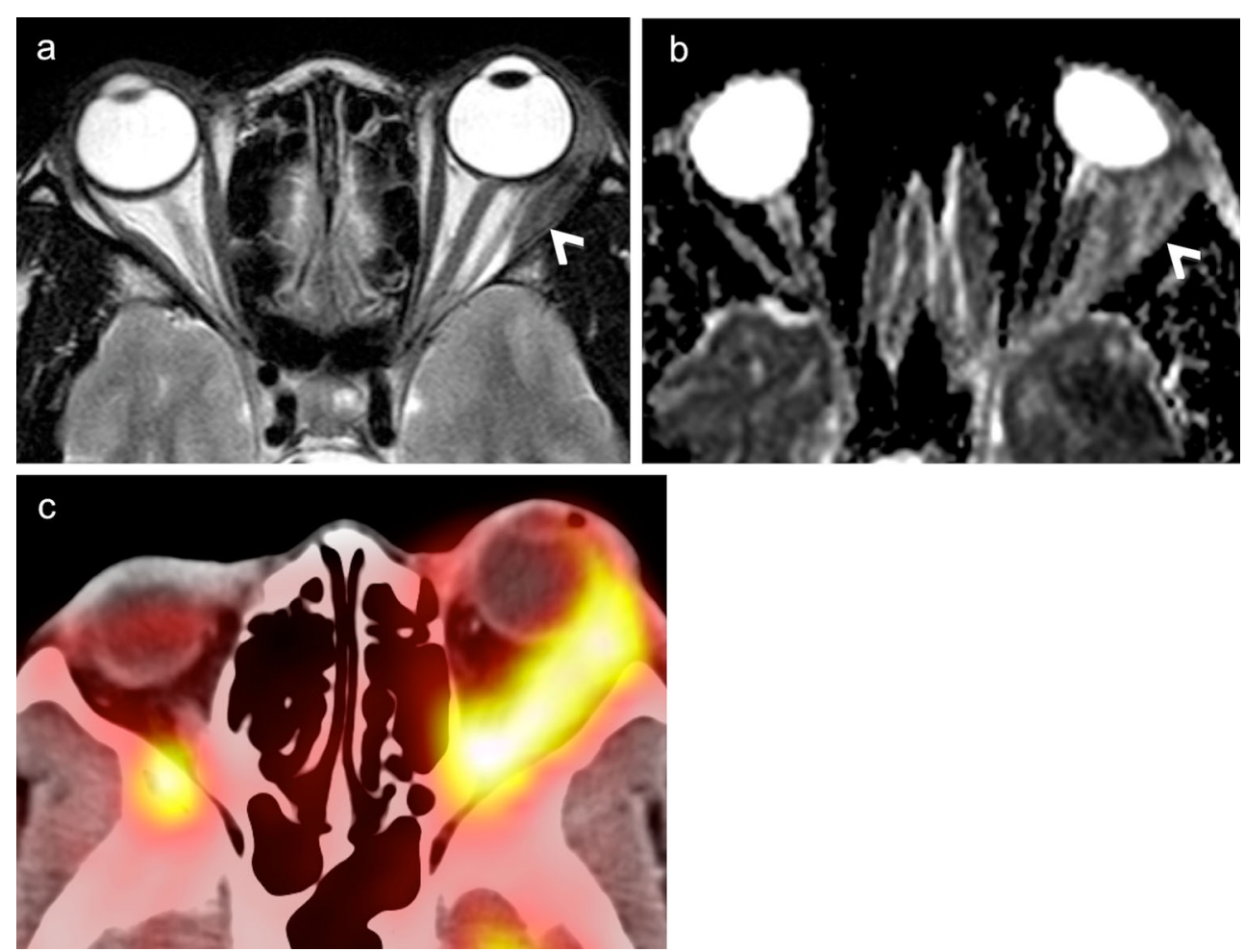

the high $\mathrm{ADC}$ values and the characteristic $\mathrm{CT}$ aspect help to avoid this pitfall.

\section{Multi-compartmental tumours}

\section{Venolymphatic malformation (VLM)}

VLM, also called lymphangioma, is a congential, hamartomous vascular malformation with variable lymphoid and venous vascular elements. It is hemodynamically isolated from systemic drainage. VLM accounts for $5 \%$ of paediatric orbital tumours; about $60 \%$ cases are diagnosed by $16-20$ years of age. It is commonly extraconal; however, it can be intraconal or multicompartmental. Proptosis, diplopia, and optic neuropathy are common presenting symptoms. Sudden increase in proptosis indicates haemorrhage within the lesion. Unlike capillary hemangiomas, which involute over time, VLM grow with the patient, especially in puberty. Treatment is usually conservative. Percutaneous sclerotherapy is performed in select cases. Surgery is difficult due to the transpatial nature and also due to risk of recurrence $[2,4,26,45,60]$.

VLM typically contain irregular shaped venous and lymphatic channels lines by flattened endothelial cells and interspersed connective tissue. The cystic spaces may show blood products or lymphatic fluid within [26, 45, 60]. VLM are usually seen on CT and MRI as poorly circumscribed, lobulated, transpatial lesions. Hemorrhagic and proteinaceous contents may appear hyperdense on NECT. Fluid-fluid levels are commonly seen. Calcification and bony erosions are uncommon. The lesion shows variable signal on T1W and T2W MR images (Fig. 17). As against capillary hemangiomas, flow voids are absent. Fat-saturated CEMRI images are ideal for mapping the lesion. Rim enhancement is commonly seen (Fig. 17) [2, 4, 26, 45, 60]. VLM show increased diffusion [13]. Their ADC values have been reported in the range of $1.44-1.53 \times 10^{-3} \mathrm{~mm}^{2} / \mathrm{s}$ and $1.75-2.2 \times 10^{-3} \mathrm{~mm}^{2} / \mathrm{s}$ $[9,12]$. On FDG PET CT, there is no focal uptake.

\section{Orbital plexiform neurofibroma (OPNF)}

OPNF is a hamartoma of neuroectodermal origin accounting for $1-2 \%$ of all orbital tumours, typically arising in the first decade of life. OPNF is diagnostic of NF-1. OPNF can involve any peripheral nerve, but the sensory nerves of the orbit are commonly involved. It is usually associated with other findings of NF-1 such as ONG, sphenoid wing dysplasia, and buphthalmos. OPNF usually presents with nodular periorbital masses, loss of vision, and proptosis. The infiltrative serpentine masses extend in both the intraconal and extraconal compartments. Progressive glaucoma, optic nerve atrophy, and blindness are eventual complications. The risk of malignant sarcomatous degeneration is about $10 \%$. ONSF are usually not amenable to surgery; however, debulking may be necessary for preservation of vision or cosmetics [4, 26, 78].

Unlike schwannomas, it is not possible to distinguish host nerve fibres separately from OPNF. The host nerve fascicles are irregularly expanded by myxoid accumulation, tumourous Schwann cells, fibroblasts, and collagen fibres [26]. Plain 
Fig. 20 50-year-old male patient with biopsy-proven IgG4-RD of the right orbit. Coronal bonewindow CT image of the orbits (a) shows a well-circumscribed, extraconal, soft tissue-density mass located inferomedially in the right orbit. It is associated with bony erosion of the lamina papyracea (arrow). b. Axial T2W image of the same patient shows very low signal within the wellcircumscribed lesion (arrow). Note that it is lower than usually seen in lymphoma. c. Axial contrast-enhanced FS TIW image shows homogeneous non-specific enhancement within the lesion (arrow). d. ADC map shows restricted diffusion $(\mathrm{ADC}=0.9 \times$ $10^{-3} \mathrm{~mm}^{2} / \mathrm{s}$ )
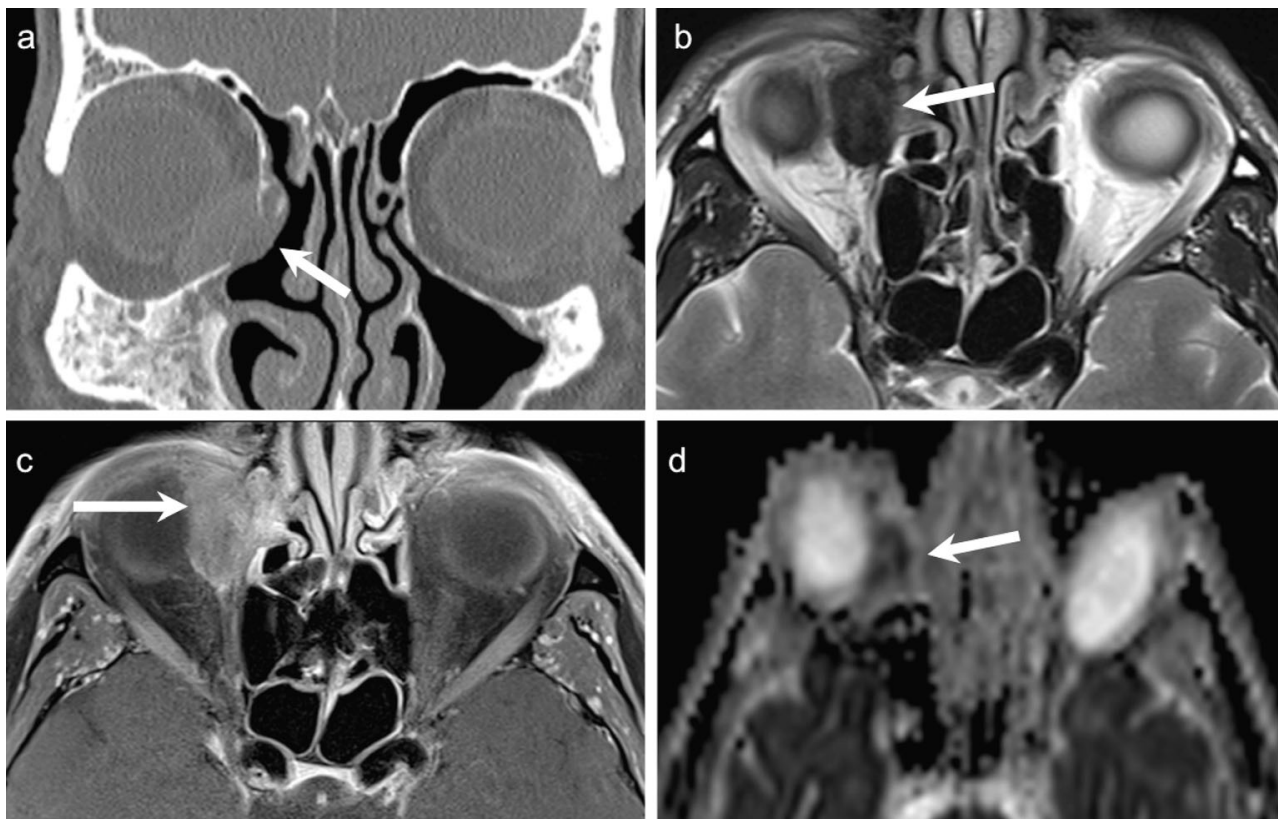
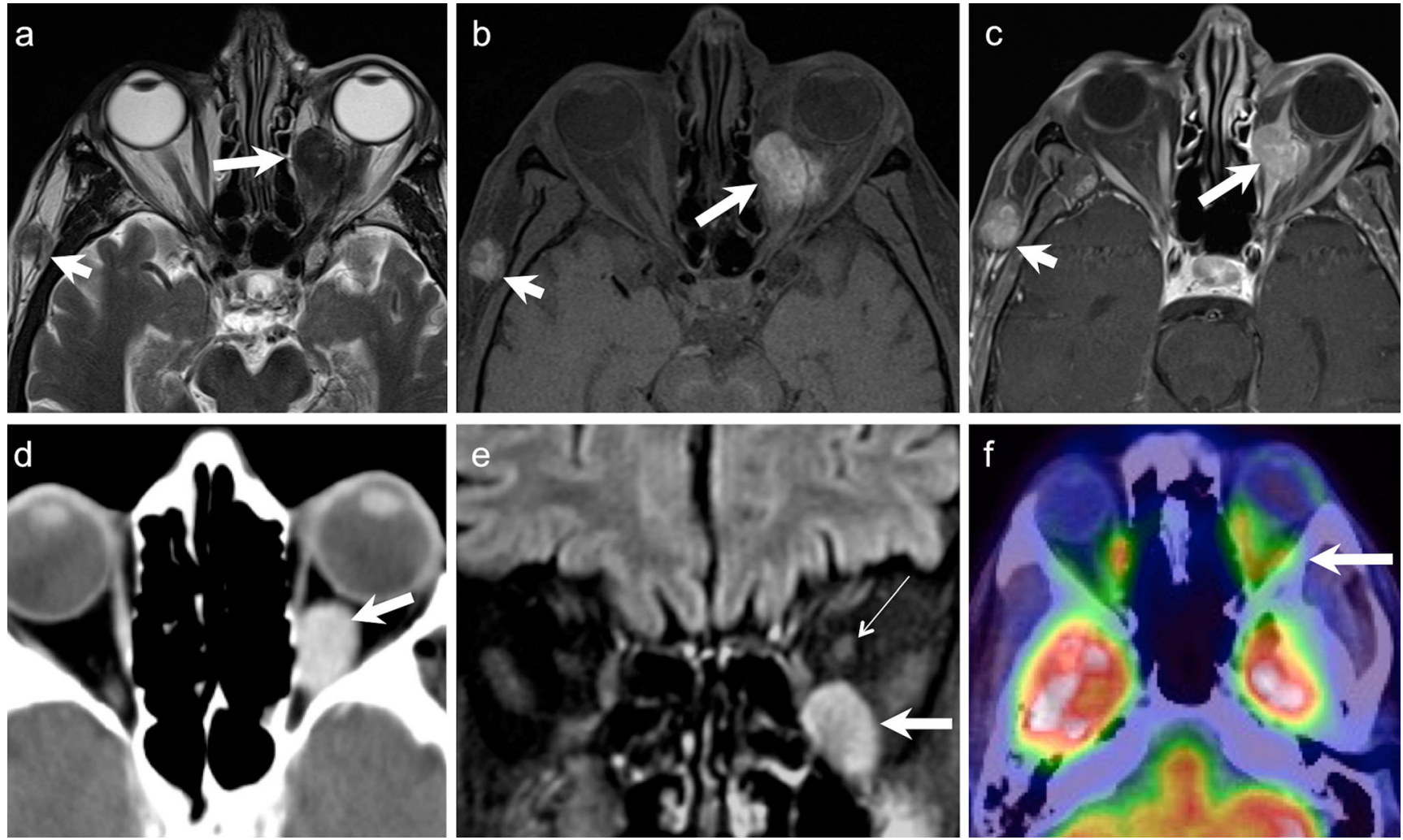

Fig. 21 Two different patients with orbital metastases. a - c. 74-year-old female patient with diplopia and a history of melanoma of the scalp 7 years previously. T2W (a), unenhanced FS T1W (b) and contrastenhanced FS T1W (c) images of the orbits shows a conal-extraconal mass in the left orbit (arrows) and a second mass with similar imaging features in the suprazygomatic right masticator space (short arrows). The strongly hyperintense signal in $b$ (arrows) suggests the presence of haemorrhage and/or melanin. Imaging findings are strongly suggestive of metastases from melanoma. Findings were confirmed by biopsy. $\mathbf{d}-\mathbf{f}$. 71-year-old female patient with known breast cancer. d. Axial CECT

image shows a well-circumscribed enhancing mass in the left orbit in extraconal location (arrow). e. Coronal STIR image of the same patient shows non-specific moderately high signal of the metastatic deposit (thick arrow). Thin arrow points to the left optic nerve. f. Axial PET/CT image of the same patient shows physiological high FDG uptake in the extraocular muscles making it difficult to detect the metastatic lesion (arrow). As the imaging findings in this case are non-specific, the diagnosis of orbital metastasis can be suggested only when the clinical background is known. Biopsy confirmed metastasis from breast cancer 

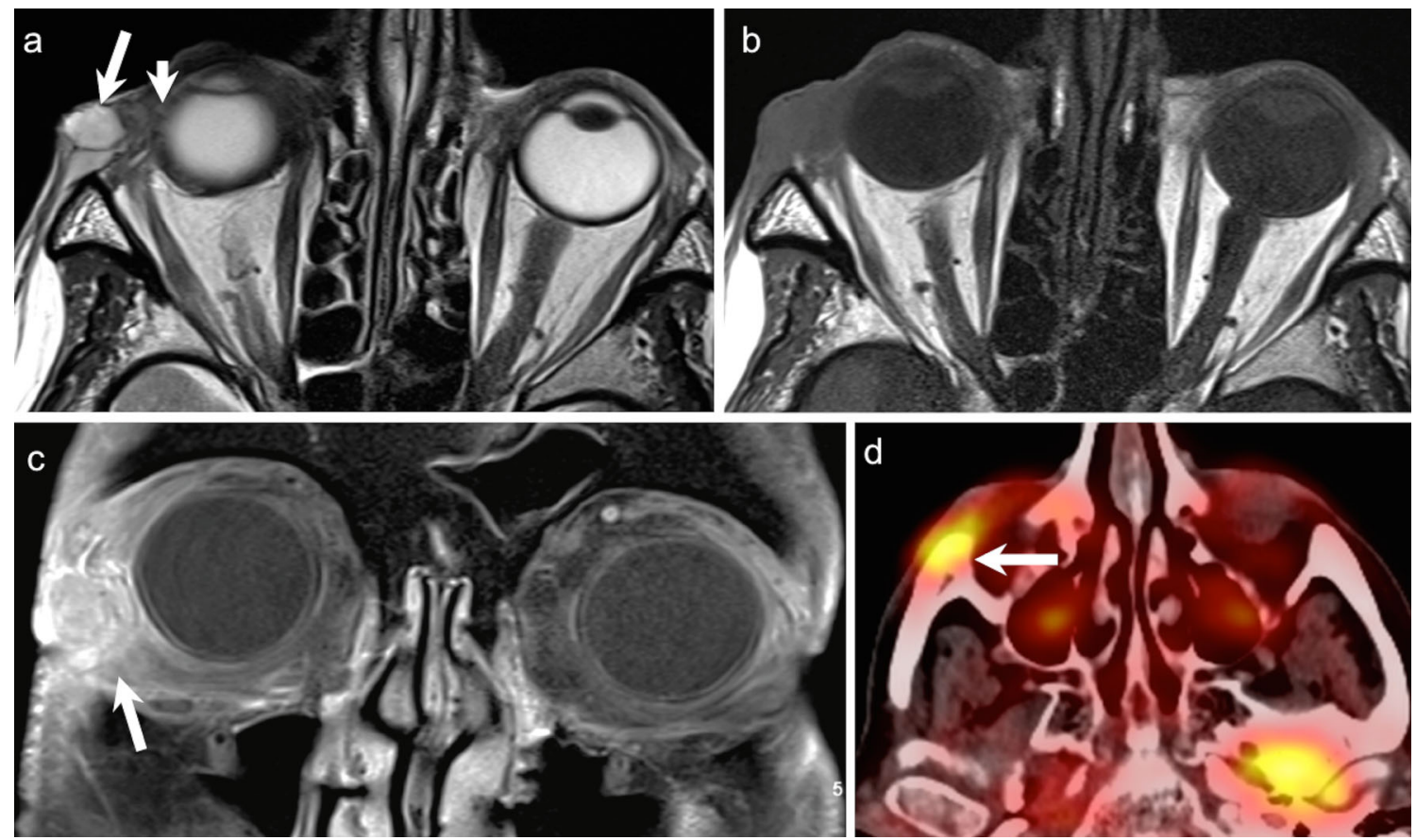

Fig. 22 60-year-old male patient with desmoplastic melanoma of the right eyelid. a. Axial T2W image shows a heterogeneous signalintensity lesion involving the subcutaneous tissue of the right lateral canthus (big arrow) with an infiltrating component extending into the extraconal compartment of the right orbit. There is suspicious invasion of the muscle cone and the globe (short arrow). b: Axial T1W image of

radiography may detect a classical defect in the greater wing of sphenoid called "Harlequin eye" appearance. Both CT and MRI show the characteristic orbital and periorbital infiltrative soft tissue masses, associated OPNF, and sphenoid wing dysplasia (Fig. 18). On T2W MRI, the nodular masses typically appear hyperintense with central low signal called the "target sign". The nodular masses show variable post contrast enhancement (Fig. 18). MRI is ideal for evaluation of the associated intracranial abnormalities. Infantile/capillary hemangioma, VLM, and RMS may mimic OPNF on imaging; however, the other associated stigmata of NFI are absent in these cases [4, 78].

OPNF show mixed or increased diffusion [13]. DTI with tractography reconstruction is increasingly being used for the pre-operative mapping of neurogenic tumours originating in the head and neck (typically from the brachial plexus), such as schwannomas and neurofibromas [79-81]. DTI with tractography reconstruction can accurately detect alterations of involved nerve fascicles, such as displacement, stretching, bowing, or rupture (Fig. 18). FDG-PET CT and more recently MRI PET are sensitive and specific tools to detect sarcomatous transformation in OPNF $[82,83]$.

\section{Inflammatory orbital pseudotumour (IOP)}

IOP is the most common cause of a painful orbital mass in adults and the third most common orbital disease after thyroid the same patient does not show hyperintense signal within the lesion in keeping with low melanin content. c. Coronal contrast-enhanced FS TIW image reveals avid enhancement within the lesion. d. Axial PET/CT image of the same patient shows high FDG uptake within the lesion due to high glucose metabolism. No other lesions were detected in the rest of the body

ophthalmopathy and lymphoproliferative disorders. IOP is a benign, non-infective, inflammatory condition without identifiable local or systemic causes and is typically seen in the 4th6th decade. The disease may present as scleritis, uveitis, lacrimal adenitis, myositis, perineuritis, or diffuse orbital inflammation. The classic clinical triad consists of unilateral orbital pain, proptosis, and impaired ocular movement. There may be associated fibrosing mediastinitis or retroperitoneal fibrosis. The diagnosis of IOP is made after exclusion of other pathologies like thyroid ophthalmopathy, lymphoma, Wegener's granulomatosis, and sarcoidosis. Treatment with steroids shows dramatic response, which helps to confirm the diagnosis $[1,2,68,84,85]$.

IOP is characterized histologically by a mixed inflammatory infiltrate, which consists of lymphocytes, plasma cells, macrophages, and eosinophilia. Fibrosis may also be seen $[84,85]$. Imaging findings may be non-specific. CT and MRI may show mass-like enhancing soft tissue within the orbit, streaky fat stranding, lacrimal gland enhancement, and optic nerve sheath enhancement (Fig. 19). The extraocular muscles are commonly enlarged and show enhancement. These inflammatory masses are usually hypointense to fat on $\mathrm{T} 1 \mathrm{~W}$ images and isohypointense on T2W images (Fig. 19). They can extend to involve the pterygopalatine fossa, nasopharynx, and cavernous sinus $[1,2,68,84,85]$. 
IOP is a great imaging mimic of several other pathologies. These include other causes of myositis (thyroid ophthalmopathy, infective cellulitis) and other T2hypointense infiltrative lesions (lymphoma, IgG4-RD, and granulomatous diseases). Thyroid ophthalmopathy causes bilateral inflammation of the extra-ocular muscles, commonly involves the inferior and medial rectus muscles, often spares the myo-tendinous junctions, and is associated with elevated thyroid-stimulating hormone levels. IgG4-RD can show similar features as thyroid ophthalmopathy, however, with normal thyroid stimulating hormone levels. Infective cellulitis is commonly associated with fever, leukocytosis, and abscess formation. Clinical features such as pain, conjunctival congestion, and eyelid oedema help to distinguish IOP from lymphoma, which commonly presents with a painless palpable mass [1, 2, 68, 84-88]. As described previously, ADC values help to accurately differentiate between IOP and lymphoma (Fig. 19) [9-12, 68]. IOP has been reported to show very high FDG uptake mimicking malignancy (Fig. 19) [89, 90].

\section{$\operatorname{IgG4}-R D$}

Immunoglobulin G4-related disease (IgG4-RD) is a chronic, systemic autoimmune, fibro-inflammatory condition. Its most common manifestation is autoimmune pancreatitis; however, retroperitoneal fibrosis, sclerosing cholangitis, interstitial nephritis, periarteritis, Riedel's thyroiditis, chronic dacryoadenitis, Mikulicz disease, and certain orbital inflammatory pseudotumours may frequently be encountered. As with IOP, IgG4-RD shows a dramatic response to corticosteroid therapy, although spontaneous resolution has been described [85-88]. Practically any part of the orbit can be involved, and the typical histological features, regardless of the affected site, are dense lympho-plasmacytic infiltration with abundant IgG4positive plasma cells, storiform-type fibrosis with obliterative phlebitis [86-88].

On MRI, IgG4-RD typically demonstrates significant hypointensity on $\mathrm{T} 1$ and $\mathrm{T} 2 \mathrm{~W}$ images with marked enhancement after contrast administration. Lymphoma shows similar MRI features on conventional sequences (Fig. 20) [86-88]. As described previously, DWI with ADC mapping may help to differentiate between the two pathologies (Fig. 20) [69]. PET CT can help to detect extra-pancreatic involvement in IgG4-RD [91].

\section{Metastases}

Metastases represent the most common orbital malignancy. Common sources for orbital metastases include melanomas, breast and lung cancers in adults, and neuroblastomas in children. Metastases commonly involve the orbital bones and extraconal compartment; however, they may also involve the choroid. Painful proptosis is the most common presentation, the exception being cirrhotic breast carcinoma metastases, where enophthalmos is a typical finding due to rectus muscle infiltration and contraction $[1,2,4]$.

Orbital metastases show histology typical to the primary tumour. High mitoses and necrosis may be seen (Fig. 21). When the primary tumour is unknown, immunohistochemistry may aid in diagnosis.

$\mathrm{CT}$ and MRI may show an enhancing infiltrative extraconal mass (Fig. 21). There can be associated bony destruction. Choroidal metastases from melanoma may appear hyperdense on NECT and hyperintense on T1W MR images. CEMRI also helps to detect associated intracranial metastases $[1,2,4]$. As described previously, metastases can mimic lymphomas on conventional CT/MR sequences. DWI can help to differentiate between the two $[9,10,12]$. An orbital metastatic deposit may be incidentally diagnosed for the first time on a PET CT. PET CT can also help to detect the primary tumour site and metastatic deposits to other organs [17-19].

\section{Lid tumours with orbital extension}

Common malignant tumours of the eyelid include squamous cell carcinoma (SCC), basal cell carcinoma, melanoma, sebaceous cell carcinoma, and lymphoma. These tumours are often highly aggressive and show contiguous invasion of orbital structures. About $43 \%$ of SCC of the eyelid show orbital involvement (Fig. 22). Perineural spread along the branches of the trigeminal nerve is also common. Orbital exenteration is required for controlling local disease spread which may be relentless and potentially fatal. Cross-sectional imaging helps in the local staging of the disease $[18,92,93]$. PET CT plays a valuable role in detecting local nodal and distant metastases from eyelid carcinomas [18, 59].

\section{Conclusion}

Evaluation of orbital masses requires a multimodality approach to balance the strength and weaknesses of each modality. Combining accurate clinical information with appropriate imaging modalities and being aware of potential diagnostic pearls and pitfalls helps to obtain the best results. Future advances in functional imaging are likely to make a significant impact on ophthalmological cancer imaging. We hope that this review has satisfactorily served the purpose of highlighting these points. 
Grants and conflict of interest information No conflicts of interest

Open Access This article is distributed under the terms of the Creative Commons Attribution 4.0 International License (http:// creativecommons.org/licenses/by/4.0/), which permits unrestricted use, distribution, and reproduction in any medium, provided you give appropriate credit to the original author(s) and the source, provide a link to the Creative Commons license, and indicate if changes were made.

\section{References}

1. Goh PS, Gi MT, Charlton A, Tan C, Gangadhara Sundar JK, Amrith S (2008) Review of orbital imaging. Eur J Radiol 66:387395

2. Heran F, Berges O, Blustajn J et al (2014) Tumor pathology of the orbit. Diagn Interv Imaging 95:933-944

3. Lee AG, Johnson MC, Policeni BA, Smoker WR (2009) Imaging for neuro-ophthalmic and orbital disease - a review. Clin Exp Ophthalmol 37:30-53

4. Rao AA, Naheedy JH, Chen JY, Robbins SL, Ramkumar HL (2013) A clinical update and radiologic review of pediatric orbital and ocular tumors. J Oncol 2013:975908

5. Varoquaux A, Rager O, Lovblad KO et al (2013) Functional imaging of head and neck squamous cell carcinoma with diffusionweighted MRI and FDG PET/CT: quantitative analysis of ADC and SUV. Eur J Nucl Med Mol Imaging 40:842-852

6. Varoquaux A, Rager O, Dulguerov P, Burkhardt K, Ailianou A, Becker M (2015) Diffusion-weighted and PET/MR Imaging after Radiation Therapy for Malignant Head and Neck Tumors. Radiographics 35(5):1502-1527

7. Becker M, Zaidi H (2014) Imaging in head and neck squamous cell carcinoma: the potential role of PET/MRI. Br J Radiol 87: 20130677

8. Becker M, Masterson K, Delavelle J, Viallon M, Vargas MI, Becker CD (2010) Imaging of the optic nerve. Eur J Radiol 74:299-313

9. Sepahdari AR, Aakalu VK, Setabutr P, Shiehmorteza M, Naheedy JH, Mafee MF (2010) Indeterminate orbital masses: restricted diffusion at MR imaging with echo-planar diffusion-weighted imaging predicts malignancy. Radiology 256:554-564

10. Sepahdari AR, Politi LS, Aakalu VK, Kim HJ, Razek AA (2014) Diffusion-weighted imaging of orbital masses: multi-institutional data support a 2-ADC threshold model to categorize lesions as benign, malignant, or indeterminate. AJNR Am J Neuroradiol 35: $170-175$

11. Fatima Z, Ichikawa T, Ishigame K et al (2014) Orbital masses: the usefulness of diffusion-weighted imaging in lesion categorization. Clin Neuroradiol 24:129-134

12. Lope LA, Hutcheson KA, Khademian ZP (2010) Magnetic resonance imaging in the analysis of pediatric orbital tumors: utility of diffusion-weighted imaging. J AAPOS 14:257-262

13. Razek AA, Elkhamary S, Mousa A (2011) Differentiation between benign and malignant orbital tumors at 3-T diffusion MR-imaging. Neuroradiology 53:517-522

14. Lober RM, Guzman R, Cheshier SH, Fredrick DR, Edwards MS, Yeom KW (2012) Application of diffusion tensor tractography in pediatric optic pathway glioma. J Neurosurg Pediatr 10:273-280

15. Zhang Y, Wan SH, Wu GJ, Zhang XL (2012) Magnetic resonance diffusion tensor imaging and diffusion tensor tractography of human visual pathway. Int J Ophthalmol 5:452-458

16. Cauley KA, Filippi CG (2013) Diffusion-tensor imaging of small nerve bundles: cranial nerves, peripheral nerves, distal spinal cord, and lumbar nerve roots-clinical applications. AJR Am J Roentgenol 201:W326-W335

17. Muzaffar R, Shousha MA, Sarajlic L, Osman MM (2013) Ophthalmologic abnormalities on FDG-PET/CT: a pictorial essay. Cancer Imaging 13:100-112

18. Hui KH, Pfeiffer ML, Esmaeli B (2012) Value of positron emission tomography/computed tomography in diagnosis and staging of primary ocular and orbital tumors. Saudi J Ophthalmol 26:365-371

19. Miyamoto J, Tatsuzawa K, Owada K, Kawabe T, Sasajima H, Mineura K (2008) Usefulness and limitations of fluorine-18fluorodeoxyglucose positron emission tomography for the detection of malignancy of orbital tumors. Neurol Med Chir (Tokyo) 48:495-499, discussion 499

20. Roe RH, Finger PT, Kurli M, Tena LB, Iacob CE (2006) Wholebody positron emission tomography/computed tomography imaging and staging of orbital lymphoma. Ophthalmology 113:1854 1858

21. Purohit BS, Ailianou A, Dulguerov N, Becker CD, Ratib O, Becker M (2014) FDG-PET/CT pitfalls in oncological head and neck imaging. Insights Imaging 5:585-602

22. Teixeira SR, Martinez-Rios C, Hu L, Bangert BA (2014) Clinical applications of pediatric positron emission tomography-magnetic resonance imaging. Semin Roentgenol 49:353-366

23. Varoquaux A, Rager O, Poncet A et al (2014) Detection and quantification of focal uptake in head and neck tumours: (18)F-FDG PET/MR versus PET/CT. Eur J Nucl Med Mol Imaging 41:462475

24. Viallon M, Cuvinciuc V, Delattre B et al (2015) State-of-the-art MRI techniques in neuroradiology: principles, pitfalls, and clinical applications. Neuroradiology 57:441-467

25. Chung EM, Specht CS, Schroeder JW (2007) From the archives of the AFIP: Pediatric orbit tumors and tumorlike lesions: neuroepithelial lesions of the ocular globe and optic nerve. Radiographics 27:1159-1186

26. Kumar V, Abbas AK, Aster JC (2014) Robbins and Cotran Pathologic Basis of Disease, 9th edn. Elsevier Saunders, Philadelphia

27. Staffieri SE, McGillivray G, Elder JE et al (2015) Managing fetuses at high risk of retinoblastoma: lesion detection on screening MRI. Prenat Diagn 35:174-178

28. Orjuela M, Castaneda VP, Ridaura C et al (2000) Presence of human papilloma virus in tumor tissue from children with retinoblastoma: an alternative mechanism for tumor development. Clin Cancer Res 6:4010-4016

29. Schueler AO, Hosten N, Bechrakis NE et al (2003) High resolution magnetic resonance imaging of retinoblastoma. $\mathrm{Br} \mathrm{J}$ Ophthalmol $87: 330-335$

30. Galluzzi P, Hadjistilianou T, Cerase A, De Francesco S, Toti P, Venturi C (2009) Is CT still useful in the study protocol of retinoblastoma? AJNR Am J Neuroradiol 30:1760-1765

31. de Graaf P, Pouwels PJ, Rodjan F et al (2012) Single-shot turbo spin-echo diffusion-weighted imaging for retinoblastoma: initial experience. AJNR Am J Neuroradiol 33:110-118

32. Radhakrishnan V, Kumar R, Malhotra A, Bakhshi S (2012) Role of $\mathrm{PET} / \mathrm{CT}$ in staging and evaluation of treatment response after 3 cycles of chemotherapy in locally advanced retinoblastoma: a prospective study. J Nucl Med 53:191-198

33. Lemke AJ, Hosten N, Bornfeld N et al (1999) Uveal melanoma: correlation of histopathologic and radiologic findings by using thinsection MR imaging with a surface coil. Radiology 210:775-783

34. McCartney AC (1995) Pathology of ocular melanomas. Br Med Bull 51:678-693

35. Erb-Eigner K, Willerding G, Taupitz M, Hamm B, Asbach P (2013) Diffusion-weighted imaging of ocular melanoma. Investig Radiol 48:702-707 
36. Reddy S, Kurli M, Tena LB, Finger PT (2005) PET/CT imaging: detection of choroidal melanoma. Br J Ophthalmol 89:1265-1269

37. Kurli M, Reddy S, Tena LB, Pavlick AC, Finger PT (2005) Whole body positron emission tomography/computed tomography staging of metastatic choroidal melanoma. Am J Ophthalmol 140:193-199

38. Jost SC, Ackerman JW, Garbow JR, Manwaring LP, Gutmann DH, McKinstry RC (2008) Diffusion-weighted and dynamic contrastenhanced imaging as markers of clinical behavior in children with optic pathway glioma. Pediatr Radiol 38:1293-1299

39. Nickerson JP, Salmela MB, Koski CJ, Andrews T, Filippi CG (2010) Diffusion tensor imaging of the pediatric optic nerve: intrinsic and extrinsic pathology compared to normal controls. J Magn Reson Imaging 32:76-81

40. Filippi CG, Bos A, Nickerson JP, Salmela MB, Koski CJ, Cauley KA (2012) Magnetic resonance diffusion tensor imaging (MRDTI) of the optic nerve and optic radiations at $3 \mathrm{~T}$ in children with neurofibromatosis type I (NF-1). Pediatr Radiol 42:168-174

41. de Blank PM, Berman JI, Liu GT, Roberts TP, Fisher MJ (2013) Fractional anisotropy of the optic radiations is associated with visual acuity loss in optic pathway gliomas of neurofibromatosis type 1. Neuro Oncol 15:1088-1095

42. Backer-Grondahl T, Moen BH, Torp SH (2012) The histopathological spectrum of human meningiomas. Int J Clin Exp Pathol 5:231242

43. Di Chiro G, Hatazawa J, Katz DA, Rizzoli HV, De Michele DJ (1987) Glucose utilization by intracranial meningiomas as an index of tumor aggressivity and probability of recurrence: a PET study. Radiology 164:521-526

44. Valotassiou V, Leondi A, Angelidis G, Psimadas D, Georgoulias P (2012) SPECT and PET imaging of meningiomas. ScientificWorldJournal 2012:412580

45. Bilaniuk LT (1999) Orbital vascular lesions. Role of imaging. Radiol Clin N Am 37(1):169-183, xi

46. Thorn-Kany M, Arrue P, Delisle MB, Lacroix F, Lagarrigue J, Manelfe C (1999) Cavernous hemangiomas of the orbit: MR imaging. J Neuroradiol 26:79-86

47. Strianese D, Napoli M, Russo C et al (2014) Coexistence of cavernous hemangioma and other vascular malformations of the orbit. A report of three cases. Neuroradiol J 27:223-231

48. Tanaka A, Mihara F, Yoshiura T et al (2004) Differentiation of cavernous hemangioma from schwannoma of the orbit: a dynamic MRI study. AJR Am J Roentgenol 183:1799-1804

49. Xian J, Zhang Z, Wang Z et al (2010) Evaluation of MR imaging findings differentiating cavernous haemangiomas from schwannomas in the orbit. Eur Radiol 20:2221-2228

50. Polito E, Burroni L, Pichierri P, Loffredo A, Vattimo AG (2005) Technetium tc $99 \mathrm{~m}$-labeled red blood cells in the preoperative diagnosis of cavernous hemangioma and other vascular orbital tumors. Arch Ophthalmol 123:1678-1683

51. Chung EM, Murphey MD, Specht CS, Cube R, Smirniotopoulos JG (2008) From the Archives of the AFIP. Pediatric orbit tumors and tumorlike lesions: osseous lesions of the orbit. Radiographics 28:1193-1214

52. Abdel Razek AA, Gaballa G, Elhawarey G, Megahed AS, Hafez M, Nada N (2009) Characterization of pediatric head and neck masses with diffusion-weighted MR imaging. Eur Radiol 19:201208

53. Jung WS, Ahn KJ, Park MR et al (2007) The radiological spectrum of orbital pathologies that involve the lacrimal gland and the lacrimal fossa. Korean J Radiol 8:336-342

54. Mafee MF, Edward DP, Koeller KK, Dorodi S (1999) Lacrimal gland tumors and simulating lesions. Clinicopathologic and MR imaging features. Radiol Clin N Am 37:219-239, xii

55. Zbaren P, Stauffer E (2007) Pleomorphic adenoma of the parotid gland: histopathologic analysis of the capsular characteristics of 218 tumors. Head Neck 29:751-757
56. Motoori K, Yamamoto S, Ueda T et al (2004) Inter- and intratumoral variability in magnetic resonance imaging of pleomorphic adenoma: an attempt to interpret the variable magnetic resonance findings. J Comput Assist Tomogr 28:233-246

57. Habermann CR, Arndt C, Graessner J et al (2009) Diffusionweighted echo-planar MR imaging of primary parotid gland tumors: is a prediction of different histologic subtypes possible? AJNR Am J Neuroradiol 30:591-596

58. Elkhamary SM (2012) Lacrimal gland lesions: Can addition of diffusion-weighted MR imaging improve diagnostic accuracy in characterization? Egypt J Radiol Nucl Med 43:165-172

59. Baek CH, Chung MK, Jeong HS et al (2009) The clinical usefulness of (18)F-FDG PET/CT for the evaluation of lymph node metastasis in periorbital malignancies. Korean J Radiol 10:1-7

60. Chung EM, Smirniotopoulos JG, Specht CS, Schroeder JW, Cube R (2007) From the archives of the AFIP: Pediatric orbit tumors and tumorlike lesions: nonosseous lesions of the extraocular orbit. Radiographics 27:1777-1799

61. Hassold N, Warmuth-Metz M, Winkler B et al (2014) Hit the mark with diffusion-weighted imaging: metastases of rhabdomyosarcoma to the extraocular eye muscles. BMC Pediatr 14:57

62. Gavane S, Cistaro A, Schoder H (2013) Utility of 18 F-FDG-PET/ CT in Soft Tissue Sarcomas. In: Cistaro A (ed) Atlas of PET/CT in Pediatric Patients. Springer, Milan, pp 87-92

63. Purohit BS, Dulguerov P, Burkhardt K, Becker M (2014) Dedifferentiated laryngeal chondrosarcoma: Combined morphologic and functional imaging with positron-emission tomography/ magnetic resonance imaging. Laryngoscope 124:E274-E277

64. Tateishi U, Hosono A, Makimoto A et al (2009) Comparative study of FDG PET/CT and conventional imaging in the staging of rhabdomyosarcoma. Ann Nucl Med 23:155-161

65. Kumar J, Seith A, Kumar A et al (2008) Whole-body MR imaging with the use of parallel imaging for detection of skeletal metastases in pediatric patients with small-cell neoplasms: comparison with skeletal scintigraphy and FDG PET/CT. Pediatr Radiol 38:953-962

66. Eckardt AM, Lemound J, Rana M, Gellrich NC (2013) Orbital lymphoma: diagnostic approach and treatment outcome. World J Surg Oncol 11:73

67. Stefanovic A, Lossos IS (2009) Extranodal marginal zone lymphoma of the ocular adnexa. Blood 114:501-510

68. Kapur R, Sepahdari AR, Mafee MF et al (2009) MR imaging of orbital inflammatory syndrome, orbital cellulitis, and orbital lymphoid lesions: the role of diffusion-weighted imaging. AJNR Am J Neuroradiol 30:64-70

69. Hiwatashi A, Yoshiura T, Togao O et al (2014) Diffusivity of intraorbital lymphoma vs. IgG4-related DISEASE: 3D turbo field echo with diffusion-sensitised driven-equilibrium preparation technique. Eur Radiol 24:581-586

70. Haradome K, Haradome H, Usui Y et al (2014) Orbital lymphoproliferative disorders (OLPDs): value of MR imaging for differentiating orbital lymphoma from benign OPLDs. AJNR Am J Neuroradiol 35:1976-1982

71. Valenzuela AA, Allen C, Grimes D, Wong D, Sullivan TJ (2006) Positron emission tomography in the detection and staging of ocular adnexal lymphoproliferative disease. Ophthalmology 113:23312337

72. Gayed I, Eskandari MF, McLaughlin P, Pro B, Diba R, Esmaeli B (2007) Value of positron emission tomography in staging ocular adnexal lymphomas and evaluating their response to therapy. Ophthalmic Surg Lasers Imaging 38:319-325

73. Platzek I, Beuthien-Baumann B, Langner J et al (2013) PET/MR for therapy response evaluation in malignant lymphoma: initial experience. MAGMA 26:49-55

74. Ginat DT, Mangla R, Yeaney G, Johnson M, Ekholm S (2012) Diffusion-weighted imaging for differentiating benign from 
malignant skull lesions and correlation with cell density. AJR Am J Roentgenol 198:W597-W601

75. Neubauer H, Evangelista L, Hassold N et al (2012) Diffusionweighted MRI for detection and differentiation of musculoskeletal tumorous and tumor-like lesions in pediatric patients. World $\mathrm{J}$ Pediatr 8:342-349

76. Su MG, Tian R, Fan QP et al (2011) Recognition of fibrous dysplasia of bone mimicking skeletal metastasis on 18F-FDG PET/CT imaging. Skelet Radiol 40:295-302

77. Stegger L, Juergens KU, Kliesch S, Wormanns D, Weckesser M (2007) Unexpected finding of elevated glucose uptake in fibrous dysplasia mimicking malignancy: contradicting metabolism and morphology in combined PET/CT. Eur Radiol 17:1784-1786

78. Jacquemin C, Bosley TM, Svedberg H (2003) Orbit deformities in craniofacial neurofibromatosis type 1. AJNR Am J Neuroradiol 24: $1678-1682$

79. Vargas MI, Gariani J, Delattre BA, Dietemann JL, Lovblad K, Becker M (2015) Three-dimensional MR imaging of the brachial plexus. Semin Musculoskelet Radiol 19:137-148

80. Vargas MI, Viallon M, Nguyen D, Delavelle J, Becker M (2010) Diffusion tensor imaging (DTI) and tractography of the brachial plexus: feasibility and initial experience in neoplastic conditions. Neuroradiology 52:237-245

81. Vargas MI, Viallon M, Nguyen D, Beaulieu JY, Delavelle J, Becker M (2010) New approaches in imaging of the brachial plexus. Eur J Radiol 74:403-410

82. Ferner RE, Golding JF, Smith M et al (2008) [18F]2-fluoro-2-deoxy-D-glucose positron emission tomography (FDG PET) as a diagnostic tool for neurofibromatosis 1 (NF1) associated malignant peripheral nerve sheath tumours (MPNSTs): a long-term clinical study. Ann Oncol 19:390-394

83. Tsai LL, Drubach L, Fahey F, Irons M, Voss S, Ullrich NJ (2012) [18F]-Fluorodeoxyglucose positron emission tomography in children with neurofibromatosis type 1 and plexiform neurofibromas: correlation with malignant transformation. J Neurooncol 108:469-475

84. Patnana M, Sevrukov AB, Elsayes KM, Viswanathan C, Lubner M, Menias CO (2012) Inflammatory pseudotumor: the great mimicker. AJR Am J Roentgenol 198:W217-W227

85. Pakdaman MN, Sepahdari AR, Elkhamary SM (2014) Orbital inflammatory disease: Pictorial review and differential diagnosis. World J Radiol 6:106-115

86. Tan TJ, Ng YL, Tan D, Fong WS, Low AS (2014) Extrapancreatic findings of IgG4-related disease. Clin Radiol 69:209-218

87. Kalapesi FB, Garrott HM, Moldovan C, Williams M, Ramanan A, Herbert HM (2013) IgG4 orbital inflammation in a 5-year-old child presenting as an orbital mass. Orbit 32:137-140

88. Sato Y, Ohshima K, Takata K et al (2012) Ocular adnexal IgG4-producing mucosa-associated lymphoid tissue lymphoma mimicking IgG4-related disease. J Clin Exp Hematop 52: $51-55$

89. Huellner MW, Schwizer B, Burger I et al (2010) Inflammatory pseudotumor of the lung with high FDG uptake. Clin Nucl Med 35:722-723

90. Alongi F, Bolognesi A, Samanes Gajate AM et al (2010) Inflammatory pseudotumor of mediastinum treated with tomotherapy and monitored with FDG-PET/CT: case report and literature review. Tumori 96:322-326

91. Nguyen VX, De Petris G, Nguyen BD (2011) Usefulness of PET/ $\mathrm{CT}$ imaging in systemic IgG4-related sclerosing disease. A report of three cases. JOP 12:297-305

92. Soysal HG, Markoc F (2007) Invasive squamous cell carcinoma of the eyelids and periorbital region. Br J Ophthalmol 91:325-329

93. Tyers AG (2006) Orbital exenteration for invasive skin tumours. Eye (Lond) 20:1165-1170 